\title{
Structure of the Algebras of Some Free Systems
}

\author{
G. F. Dell'Antonio \\ Istituto di Fisica Teorica, Università di Napoli \\ Istituto Nazionale di Fisica Nucleare Sezione di Napoli
}

Received November 14, 1967

\begin{abstract}
We give a detailed analysis of the structure of some Von Neumann algebras which describe free relativistic fields or infinite systems of free particles with finite density.
\end{abstract}

\section{Introduction}

We intend to give the explicit structure of Von Neumann algebras which have been used in the description of some physical systems.

Some of these algebras are associated to an infinite free Bose or Fermi gas at any temperature $[1,2]$; others to relativistic free fields described in terms of observables associated to each space-type region [3, 4].

The purpose of the analysis we are going to make, is twofold. One will gain a better understanding of some (formal) properties of infinite free systems with finite density, and will be able to give a completely algebraic description of a free field.

Also, one may hope that part of the structure exhibited for the particular cases at hand, has a general character (i.e. is shared by less trivial systems) and may be used as a first step in the construction of more realistic models.

We shall follow the good practice of going from the simpler to the more complicated, and shall therefore begin with a simple example, i.e. the free Fermi gas at finite temperature.

\section{Free Fermi Gas at Finite Temperature}

The general properties of this system have been analyzed in ref. (2). It is shown there that an infinite free Fermi gas can be described in terms of a suitable representation of the canonical anti-commutation relation (c.a.r.). By representation of the c.a.r. on a Hilbert space $\mathscr{H}$ we mean a set $a_{i}$ of operators on $\mathscr{H}$ such that

$$
\left[a_{i}, a_{j}\right]_{+}=0, \quad\left[a_{i}, a_{j}^{*}\right]_{+}=\delta_{i j}
$$

where $[c, d]_{+}=c d+d c$ and $c^{*}$ denotes the adjoint of $c$. A representation is called irreducible if the smallest Von Neumann algebra [5]"which containes all the $a_{i}$, $a_{i}^{*}$ (i.e. the Von Neumann algebra generated by the $a_{i}^{\prime}$ 's) is $\mathscr{B}(\mathscr{H})$, the set of all bounded operators on $\mathscr{H}$.

6 Commun. math. Phys., Vol. 9 
Two representations on $\mathscr{H}^{(1)}, \mathscr{H}^{(2)}$ are equivalent if there exists an isometry of $\mathscr{H}^{(1)}$ onto $\mathscr{H}^{(2)}$ such that the induced isomorphism of $\mathscr{B}\left(\mathscr{H}_{1}\right)$ on $\mathscr{B}\left(\mathscr{H}_{2}\right)$ carries $a_{i}^{(1)}$ to $a_{i}^{(2)}$ for all $i$.

A representation is of Fock type iff there exists in $\mathscr{H}$ a vector $\Omega$ with the properties

$$
a_{i} \Omega=0 \quad \forall i
$$

Let $\left\{a(f) ; f \in \mathscr{L}_{r}^{2}\left(\mathbf{R}^{n}\right)\right\}$ define on $\mathscr{H}$ a Fermi system [6] on $\mathscr{L}^{2}\left(\mathbf{R}^{n}\right)$. This is a linear mapping $\mathscr{L}^{2}\left(\mathbf{R}^{n}\right) \ni f \rightarrow \underline{a}(f) \in \mathscr{B}(\mathscr{H})$ which satisfies

$$
\left[\underline{a}(f), \underline{a}^{*}(g)\right]_{+}=(f, g), \quad[\underline{a}(f), \underline{a}(g)]_{+}=0
$$

where $(f, g)$ is the scalar product in $\mathscr{L}^{2}\left(\mathbf{R}^{n}\right)$. If this representation of the c.a.r. is of Fock type, we shall write $\mathscr{H} \equiv \mathscr{H}_{F}^{a}$. Let $\{\underline{b}(f)\}$ define another such system and set ${ }^{1}$

$$
\mathscr{K}=\mathscr{H} \frac{a}{F} \otimes \mathscr{H} \frac{b}{F} .
$$

There are canonical isomorphic maps $\varphi_{a}$, resp. $\varphi_{b}$, of $\mathscr{B}\left(\mathscr{H} \frac{a}{F}\right)$, resp. $\mathscr{B}\left(\mathscr{H} \frac{b}{F}\right)$, on $\mathscr{B}(\mathscr{K})$ with the properties

$$
\left[\varphi(\underline{a}(f)), \varphi\left(\underline{a}^{*}(g)\right)\right]_{+}=(f, g), \quad\left[\varphi(\underline{b}(f)), \varphi\left(\underline{b}^{*}(g)\right)\right]_{+}=(f, g)
$$

all other anti-commutators vanish.

This isomorphism can be explicitly given through

$$
\begin{aligned}
& \underline{a}(f) \stackrel{\varphi_{a}}{\longrightarrow} \underline{a}(f) \otimes \mathbf{I} \\
& \underline{b}(f) \stackrel{\varphi_{b}}{\longrightarrow} e^{i \pi N(a)} \otimes \underline{b}(f) .
\end{aligned}
$$

Here $N(\underline{a})=\sum_{k} \underline{a}^{*}\left(f_{k}\right) \underline{a}\left(f_{k}\right)$, with $\left\{f_{k}\right\}$ an orthonormal basis of $\mathscr{L}^{2}\left(\mathbf{R}^{n}\right)$. $N(\underline{a})$ exists and is independent of the basis used in its definition [6].

We shall write $a(f) \equiv \varphi(\underline{a}(f)), \quad b(f) \equiv \varphi(\underline{b}(f))$.

It is clear that $\{a(f)\}$, resp. $\{b(f)\}$ define $\mathscr{K}$ a representation of the Fermi system which is a multiple of the Fock one. We shall use the notation $\mathscr{K} \equiv \mathscr{H}_{F}^{a, b}$. With these preliminaries out of way, we can state the results of ref. (2) as follows:

A Fermi gas at finite temperature (in an $n$-dimensional space), with no condensation and with density distribution $\varrho(k), k \in \mathbf{R}^{n}$ (in "momentum" space), can be described by the Von Neumann algebra on $\mathscr{H}_{\boldsymbol{F}}^{a, b}$ generated by the representation of the c.a.r. given by operators $\alpha, \alpha^{*}$ of the form

$$
\begin{aligned}
\alpha(f) & =a(\sqrt{1-\varrho} f)-b *(\sqrt{\varrho} f) \\
\alpha^{*}(f) & =a^{*}(\sqrt{1-\varrho f})-b(\sqrt{\varrho} f)
\end{aligned}
$$

(one has $0 \leqq \varrho \leqq 1$ ).

${ }^{1}$ For definitions and basic properties of tensor product of Hilbert spaces and of the Von Neumann algebras associated with them we shall refer to [7]. 
By definition, there exists in $\mathscr{H}_{F}^{a, b}$ a vector $\Omega_{0}$ such that

$$
a(f) \Omega_{0}=b(f) \Omega_{0}=0 \quad \forall f \in \mathscr{L}^{2}\left(\mathbf{R}^{n}\right)
$$

The representation given by (1) is not in general of Fock type. We shall denote by $\mathscr{A}$ the Von Neumann algebra it generates ${ }^{2}$. Another representation of the c.a.r. which will be of interest is the one given on $\mathscr{H}_{F}^{a, b}$ by the operators

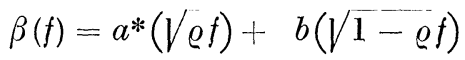

$$
\begin{aligned}
& \beta^{*}(f)=a(\sqrt{\varrho} f)+b^{*}(\sqrt{1-\varrho} f) \text {. }
\end{aligned}
$$

The corresponding algebra will be denoted by $\mathscr{B}$.

One easily checks that

$$
[\alpha(f), \beta(g)]_{+}=\left[\alpha(f), \beta^{*}(g)\right]_{+}=0 \quad \forall f, g \in \mathscr{L}^{2}\left(\mathbf{R}^{n}\right) .
$$

It is shown in ref. (2) that $\mathscr{B}$ is unitarily equivalent to $\mathscr{A}$ and that $\mathscr{A} \cap \mathscr{B}=\{c \cdot \mathbf{l}\}$ (the multiples of the identity operator on $\mathscr{K}_{\vec{i}}^{a, b}$ ); it is also shown that $\mathscr{A}$ is a factor, i.e. that $\mathscr{A} \cap \mathscr{A}^{\prime}=\{c \cdot \mathbf{I}\}$ where $\mathscr{A}^{\prime}$ is the commutant of $\mathscr{A}$. We shall re-obtain these results as a by-product of our structure analysis; we shall also give the connection between $\mathscr{A}^{\prime}$ and $\mathscr{B}$.

\section{Preliminary Results}

To study the structure of the algebras $\mathscr{A}$ and $\mathscr{B}$ we shall use the following :

Lemma $1[8]^{3}$. Let $A$ be a self-adjoint operator on a separable Hilbert space $\mathscr{H}$. One can find on $\mathscr{H}$ two self-adjoint operators $D$ and $T$, where $D$ has totally discrete spectrum and $T$ is a Hilbert-Schmidt operator (i.e. trace $\left.T^{*} T<\infty\right)$, such that

$$
A=D+T \text {. }
$$

Moreover, $D$ can be so chosen that $D=A$ on the subspace associated to the discrete spectrum of $A$; and that the spectrum of $D$ is dense in the continuous part of the spectrum of $A$.

We shall use this lemma in the following way. Let $\mathscr{N}$ be the subspace of $\mathbf{R}^{n}$ on which $\varrho$ takes the value $1, \mathscr{L}^{2}(\mathscr{N})$ the corresponding subspace of $\mathscr{L}^{2}\left(\mathbf{R}^{n}\right)$. If $\mathscr{N}$ has Lebesque measure zero, $\mathscr{L}^{2}(\mathscr{N})$ is empty.

We take for $A$ the null operator on $\mathscr{L}^{2}(\mathscr{N})$ and, on $\mathscr{L}^{2}\left(\mathbf{R}^{n}\right) \ominus \mathscr{L}^{2}(\mathscr{N})$ the operator defined by multiplication by $\sqrt{\frac{\varrho}{1-\varrho}}$. Let $f_{i} \in \mathscr{L}^{2}\left(\mathbf{R}^{n}\right), \lambda_{i} \in \mathbf{R}^{1}$, $i \in I$ ( $I$ the positive integers) be the eigenvalues and eigenvectors of $D$.

${ }^{2}$ If condensation is present, the algebra of interest is not $\mathscr{A}$ but $\mathscr{A} \otimes \mathscr{J}$, where $\mathscr{J}$ is a type I factor.

${ }^{3}$ We include for completeness in an appendix the proof of this lemma. 6* 
The lemma states that $A-D$ is Hilbert-Schmidt, i.e.

$$
\begin{aligned}
\infty>\sum_{i}\left(f_{i},(A-D)^{2} f_{i}\right) & =\sum_{i}^{\prime}\left(f_{i},\left(A-\lambda_{i}\right)^{2} f_{i}\right) \\
& =\sum_{i}^{\prime}\left|\left(\sqrt{\frac{\varrho}{1-\varrho}}-\lambda_{i}\right) f_{i}\right|^{2}
\end{aligned}
$$

where $\Sigma^{\prime}$ is the sum extended to all $i$ 's for which $f_{i} \notin \mathscr{L}^{2}(\mathscr{N})$. Since $\sqrt{1-\varrho}$ is a bounded operator, one has from (5)

$$
\left.\sum_{i}\left(\sqrt{\varrho}-\lambda_{i} \sqrt{1-}-\overline{0}\right) f_{i}\right|^{2}<\infty
$$

Recall that $\mathscr{A}, \mathscr{B}$ are generated by operators of the form

and define

$$
\begin{aligned}
& \alpha(f)=a(\sqrt{1-\varrho f})-b *(\sqrt{\varrho} f), \\
& \beta(f)=a^{*}(\sqrt{\varrho} f)+b(\sqrt{1-\varrho f})
\end{aligned}
$$

$$
\begin{aligned}
& A_{i}=\frac{1}{\sqrt{1+\lambda_{i}^{2}}}\left[a\left(\sqrt{1-\varrho} f_{i}+\lambda_{i} \sqrt{\varrho} f_{i}\right)+b^{*}\left(\lambda_{i} \sqrt{1-\varrho} f_{i}-\sqrt{\varrho} f_{i}\right)\right] \\
& B_{i}=\frac{1}{\sqrt{1+\lambda_{i}^{2}}}\left[a^{*}\left(-\sqrt{\varrho} f_{i}+\lambda_{i} \sqrt{1-\varrho} f_{i}\right)+b\left(\lambda_{i} \sqrt{\varrho} f_{i}+\sqrt{1-\varrho} f_{i}\right)\right] .
\end{aligned}
$$

For notational compactness we have taken $1 / \lambda_{i}=0$ (i.e. $A_{i}=a\left(f_{i}\right)$ ) if $f_{i} \in \mathscr{L}^{2}(\mathscr{N})$. One can easily convince oneself that the $A_{i}, B_{i}$ define on $\mathscr{H}_{F}^{a, b}$ an irreducible representation of a Fermi system over

$$
\mathscr{L}^{2}\left(\mathbf{R}^{n}\right) \oplus \mathscr{L}^{2}\left(\mathbf{R}^{n}\right) .
$$

We claim that this representation is of Fock type; this is equivalent to saying that there exists in $\mathscr{H}_{F}^{a, b}$ a cyclic vector with the properties

In fact, let

$$
A_{i} \Omega=B_{i} \Omega=0 \quad \forall i \in I .
$$

$$
\begin{aligned}
& A_{i}^{N} \equiv \begin{cases}A_{i}, \quad i \leqq N \\
\frac{1}{\sqrt{1+\lambda_{i}^{2}}}\left[a\left(\left(\sqrt{1-\varrho}+\lambda_{i} \sqrt{\varrho}\right) f_{i}\right)+b\left(\left(\lambda_{i} \sqrt{1-\varrho}-\sqrt{\varrho}\right) f_{i}\right)\right] & i>N\end{cases} \\
& B_{i}^{N} \equiv \begin{cases}B_{i}, \quad i \leqq N & (9) \\
\frac{1}{\sqrt{1+\lambda_{i}^{2}}}\left[a\left(\left(-\sqrt{\varrho}+\lambda_{i} \sqrt{1-\varrho}\right) f_{i}\right)-b\left(\left(\lambda_{i} \sqrt{\varrho}+\sqrt{1-\varrho}\right) f_{i}\right)\right] & i>N .\end{cases}
\end{aligned}
$$

For each $N,\left\{A_{i}^{N}, B_{i}^{N}\right\}$ define on $\mathscr{H}_{F}^{a, b}$ an irreducible representation of the c.a.r. of Fock type. Indeed, $A_{i}^{(N)} \Omega_{0}=B_{i}^{(N)} \Omega_{0}=0$ for $i>N$ (see eq. (2)) and all representations of a Fermi system over a finite-dimensional space are equivalent. There exists therefore $\Omega^{N} \in \mathscr{H}_{F}^{a, b}$, with the properties

$$
A_{i}^{N} \Omega^{N}=B_{i}^{N} \Omega^{N}=0, \quad \forall i
$$


One now checks that inequality (6) guarantees the convergence of the sequence $\Omega^{N}, N \rightarrow \infty$.

In fact,

$$
\begin{aligned}
& \lim _{\substack{M, N \rightarrow \infty \\
N>M}}\left|\Omega^{N}-\Omega^{M}\right|^{2} \\
& =2 \lim \left(1-\exp \left[\frac{1}{2} \sum_{n=M}^{N} \log \left(1-\frac{1}{\left(1+\lambda_{i}^{2}\right)}\right)\left|\left(\lambda_{i} \sqrt{1-\varrho}-\sqrt{\varrho}\right) f_{i}\right|^{2}\right]\right)=0 .
\end{aligned}
$$

Let $\Omega$ be the limit vector. For every $i \in I$, one has

$$
B_{i} \Omega=A_{i} \Omega=0 \text {. }
$$

Indeed, e.g.

$$
\left|B_{i} \Omega\right|=\lim _{N \rightarrow \infty}\left|B_{i} \Omega^{N}\right|=\lim _{N \rightarrow \infty}\left|B_{i}^{N} \Omega^{N}\right|=0
$$

where the first equality follows from $\left\|B_{i}\right\|=1$, the second from Eq. (9), and the third from Eq. (10).

Since the representation defined by $A_{i}, B_{i}$ on $\mathscr{H}_{F}^{a, b}$ is of Fock type, one can write ${ }^{4}$

$$
\mathscr{H}_{F}^{a, b}=\mathscr{H}_{F}^{A, B}=\bigotimes_{i}^{\Omega}\left(\mathscr{H}_{F}^{A_{i}} \otimes \mathscr{H}_{F}^{B_{i}}\right)
$$

where $\Omega=\bigotimes_{i}\left(\Omega_{i}^{A} \otimes \Omega_{i}^{B}\right)$, and there are canonical isomorphisms $A_{i} \rightarrow \underline{A}_{i} \in \mathscr{B}\left(\mathscr{H}_{F}^{A_{i}}\right), \quad B_{i} \rightarrow B_{i} \in \mathscr{B}\left(\mathscr{H}_{F}^{B_{i}}\right)$ such that $\underline{A}_{i} \Omega_{i}^{A}=B_{i} \Omega_{i}^{B}=0$. Notice that $\mathscr{H}_{F}^{A_{i}} \otimes \mathscr{H}_{F}^{B_{i}}$ is a four-dimensional space. From Eqs. (7), (3) one obtains

$$
\begin{aligned}
& \alpha\left(f_{i}\right) \equiv \alpha_{i}=\frac{1}{\sqrt{1+\lambda_{i}^{2}}} A_{i}+\frac{\lambda_{i}}{\sqrt{1+\lambda_{i}^{2}}} B_{i}^{*} \\
& \beta\left(f_{i}\right) \equiv \beta_{i}=\frac{1}{\sqrt{1+\lambda_{i}^{2}}} B_{i}-\frac{\lambda_{i}}{\sqrt{1+\lambda_{i}^{2}}} A_{i}^{*} .
\end{aligned}
$$

\section{Structure Analysis}

All finite-dimensional representations of the c.a.r. being unitarily equivalent, one has

where $\Omega_{i}=\Omega_{i}^{A} \otimes \Omega_{i}^{B}$.

$$
\mathscr{H}_{F}^{A}, B=\bigotimes_{i}^{\otimes \Omega_{i}}\left(\mathscr{H}_{F^{\prime}, i}^{\boldsymbol{\gamma}} \otimes \mathscr{H}_{F, i}^{\beta}\right)
$$

One can now find a canonical isomorphism

$$
\alpha_{i} \rightarrow \underline{\alpha}_{i} \equiv \mathbf{I}_{1} \otimes \cdots \otimes\left(\alpha_{i}^{\prime} \otimes \mathbf{I}_{i}^{\beta}\right) \otimes \mathbf{I}_{i+1} \otimes \cdots
$$

${ }^{4}$ See Ref. [7] or [9] for the definition and properties of a separable Hilbert space $\mathscr{H}$, infinite direct tensor product of Hilbert spaces $\mathscr{H}_{2}$ relative to a direct product vector $\Omega=\otimes \Omega_{i}, \Omega_{i} \in \mathscr{H}_{i}$. We shall only recall that, roughly speaking, $\mathscr{H}$ is the closure of the set of those vectors, in the infinite tensor product space, which have $i^{\text {th }}$ component $\neq \Omega_{i}$ only for a finite set of values of the index $i$. 
where $\alpha_{i}^{\prime}$ give a representation of the c.a.r. on $\mathscr{H}_{F}^{\alpha_{i}}$ and $\mathbf{I}_{i}$ is the identity on $\mathscr{H}_{F}^{\beta_{i}}$.

The algebra $\mathscr{A}$ generated by the $\underline{\alpha}_{i}$ can be identified with $\mathscr{A}$, the algebra generated by the $\alpha_{i}$; indeed, the isomorphism can be written

$$
\underline{\alpha}_{i}=\bigcup_{i} \alpha_{i}, \quad \bigcup_{i}=\prod_{i} e^{i \pi N_{k}^{(\alpha)}}, \quad N_{k}^{(\alpha)}=\alpha_{k}^{*} \alpha_{k}
$$

and the double inclusion $\mathscr{A} \subset \mathscr{A} \subset \mathscr{A}$ is evident from Eq. (15) and $\alpha_{i}^{*} \alpha_{i}=\underline{\alpha}_{i}^{*} \underline{\alpha}_{i}$. Since $\alpha_{i}^{\prime}$ acts irreducibly on $\mathscr{H}_{F}^{\alpha_{i}}$ for each $i$, the algebra ( $\mathscr{A}$ and therefore) $\mathscr{A}$ has the form

$$
\mathscr{A}=\left[\mathbf{I}_{i} \otimes \cdots \otimes\left(\mathscr{B}\left(\mathscr{H}_{F}^{\alpha_{i}}\right) \otimes \mathbf{I}_{i}^{\beta}\right) \otimes \mathbf{I}_{i+1} \otimes \cdots ; i=1,2, \ldots\right]^{-}
$$

where $[\mathscr{M}]$ - indicates the Von Neumann algebra generated by the set $\mathscr{M}$ of operators. $\mathscr{A}$ is therefore [7] simple (it has no two-sided ideals).

It also follows [10] from (16) that $\mathscr{A}$ is a factor and that $\mathscr{A}$ is unitarily equivalent to its commutants $\mathscr{A}^{\prime}$. The factor $\mathscr{A}^{\prime}$ is given explicitely by

$$
\mathscr{A}^{\prime}=\left[\mathbf{I}_{1} \otimes \cdots \otimes\left(\mathbf{I}_{i}^{\alpha} \otimes \mathscr{B}\left(\mathscr{H}_{F}^{\beta_{i}}\right)\right) \otimes \mathbf{I}_{i+1} \otimes \cdots ; i=1,2, \ldots\right]^{-} .
$$

The type of the factor $\mathscr{A}$ is determined by the sequence $\left\{d_{k}\right\}$ where

$$
d_{i}=\inf _{\xi_{i}, \eta_{i}}\left|\Omega-\xi_{i} \otimes \eta_{i}\right|, \quad \xi_{i} \in \mathscr{H}_{F^{*}}^{\alpha_{i}} \quad \eta_{i} \in \mathscr{H}_{F^{*}}^{\beta_{i}}, \quad\left|\xi_{i}\right|=\left|\eta_{i}\right|=1 .
$$

One verifies without difficulty that in our case

$$
d_{i}=2\left(1-\sup \left[\frac{1}{\sqrt{1+\lambda_{i}^{2}}}, \frac{\lambda_{i}}{\sqrt{1+\lambda_{i}^{2}}}\right]\right)
$$

(notice that $d_{i} \leqq \sqrt{2}(\sqrt{2}-1)$ and $d_{i}=\sqrt{2}(\sqrt{2}-1)$ iff $\lambda_{i}=1$ ).

The type of the factor $\mathscr{A}$ is now determined by the following well known

Lemma $2[7,11]$. Let $\left\{d_{i}\right\}$ be an infinite sequence of positive numbers with exactly one accumulation point $d_{\infty}$. Then the factor $\mathscr{A}$ as defined in (16) is

1) of type I if $\sum_{i} d_{i}<\infty, d_{\infty}=0$,

2) of type II if $\sum_{i}\left|\sqrt{2}(\sqrt{2}-1)-d_{i}\right|<\infty, d_{\infty}=\sqrt{2}(\sqrt{2}-1)$,

3) of type III otherwise.

We shall need two more results:

If $\mathscr{A}$ and $\mathscr{B}$ are factors, one proves [5] that $\mathscr{A} \otimes \mathscr{B}$ is type II if $\mathscr{A}$ is type II and $\mathscr{B}$ is type I or II, and that $\mathscr{A} \otimes \mathscr{B}$ is type III if $\mathscr{A}$ is type III.

Finally, if $\mathscr{M}_{i}$ are Von Neumann algebras, one proves [10] that $\left[\mathbf{I}_{1} \otimes \cdots \otimes \mathscr{M}_{i} \otimes \mathbf{I}_{i+1} \otimes \cdots, i \in I\right]^{-}$ $=\left[\mathbf{I}_{k_{1}} \otimes \cdots \otimes \mathscr{M}_{k_{i}} \otimes \mathbf{I}_{k_{i+1}} \otimes \cdots, k_{i} \in K\right]^{-} \otimes\left[\mathbf{I}_{h_{1}} \otimes \cdots \otimes \mathscr{M}_{h_{1}} \otimes \cdots, h_{i} \in H\right]^{-}$ if $K \cap H=\emptyset, K+H=I$ ( $I$, a denumerable index set). 
As we have already remarked, $\mathscr{L}^{2}\left(D_{i}\right)$ is either zero or infinitedimensional, where $D_{i}$ is defined by $D_{i}:\left\{k: \varrho(k)=\lambda_{i}\right\}$. We can now collect all the information we have obtained so far on the structure of $\mathscr{A}$, in the following

Proposition 1. a) The factor $\mathscr{A}$ associated to the description of a Fermi gas at finite temperature is

- of type $I$ if the density function $\varrho(k)$ takes values different from 0,1 only on a set of (Lebesgue) measure zero,

- of type $I I$ if $\varrho(k)$ takes values different from $0,1,1 / 2$ only on a set of measure zero, and the value $1 / 2$ is taken on a set of non-zero measure,

- of type III otherwise.

b) Moreover, two algebras $\mathscr{A}_{1}$ and $\mathscr{A}_{2}$ relative to distribution functions $\varrho_{1}$ and $\varrho_{2}$ are unitarily equivalent (i.e. spatially isomorphic) if they are algebraically isomorphic.

c) $\mathscr{A}_{1}$ and $\mathscr{A}_{2}$ are isomorphic if $\underline{\varrho}_{1}$ and $\underline{\varrho}_{2}$ have the same continuous spectrum independently of multiplicity and their discrete isolated eigenvalues $\varrho_{1}^{(i)}, \varrho_{2}^{(i)}$, coincide (again discarding multiplicity). Here

$\underline{\varrho}_{k}=\inf \left[\varrho_{k}, 1-\varrho_{k}\right], k=1,2$.

Part $\mathrm{C}$ of proposition 1 requires some comments.

The isomorphism between $\mathscr{A}_{1}$ and $\mathscr{A}_{2}$, when it holds, is independent of the multiplicity of the spectra of $\varrho_{1}, \varrho_{2}$. For the isolated point spectrum this follows because, as we have seen, the multiplicity is always infinite there. For the continouum part of the spectrum, independence from multiplicity is guaranteed by the following facts: the corresponding algebra $\mathscr{A}_{c}$ is always of type III; $\mathscr{A}_{c} \otimes \mathscr{B}\left(\mathscr{H}_{N}\right)$ is algebraically isomorphic to $\mathscr{A}_{c}\left(N=1,2, \ldots ; \mathscr{H}_{k}\right.$ an $N$-dimensional Hilbert space); algebraic isomorphism implies spatial isomorphism for type III factors on a separable Hilbert space [5] (the latter result is also used in statement b) of the Proposition). The conditions for isomorphism given in c) are sufficient; for the discrete isolated part of the spectrum this follows from the construction explicitely given for $\mathscr{A}$ (Eq. 16). The same arguments can be used for the continuous part of the spectrum, if one remarks that, when $\sum_{c}\left(\underline{\varrho}_{1}\right)=\sum_{c}\left(\underline{\varrho}_{2}\right)\left(\sum_{c}(\varrho)\right.$ is the continuous part of the spectrum of $\varrho$ ), one can choose in Lemma 1 the same spectrum for $D_{1}$ and $D_{2}{ }^{5}$.

Notice also that the points of the discrete spectrum which are not isolated from the continuous part, do not enter in the conditions for the isomorphism between $\mathscr{A}_{1}$ and $\mathscr{A}_{2}$. In fact, if $a_{0}$ is a discrete non-isolated point in the spectrum of $A$, one can find a sequence of points $\left\{a_{i}\right\}$, which belong to the continuous part of the spectrum of $A$ and to the spectrum

${ }^{5}$ It seems quite probable that the conditions stated in c) be also necessary. One would need for the proof a refinement of recent results by R. Powers [12]; we hope to come back in the future on this point. 
of $D$ (Lemma 1) such that $\sum_{i}\left|a_{0}-a_{i}\right|<\infty$. Since for the continuous part of the spectrum of $A$ the isomorphism is independent of multiplicity, the statement follows.

This implies in particular that, if $\varrho_{1}, \varrho_{2}$ are continuous functions, and if they have the same range, the two factors $\mathscr{A}_{1}$, and $\mathscr{A}_{2}$ are spatially isomorphic (and the corresponding representations of the c.a.r. are equivalent).

We come back now to the factors $\mathscr{A}, \mathscr{B}$ associated to an infinite Fermi system with density function $\varrho(k)$.

We have already seen the structure of $\mathscr{A}$.

The algebra $\mathscr{B}$ is spatially isomorphic to $\mathscr{A}$; this can be seen using Proposition 1, or also directly as follows.

The algebraic automorphism $\phi$ of $[\mathscr{A} \cup \mathscr{B}]=$ (the smallest $C^{*}$ algebra which contains $\mathscr{A}$ and $\mathscr{B}$ ) defined by

$$
\alpha_{k} \stackrel{\phi}{\longrightarrow} \beta_{k}, \quad \beta_{k} \stackrel{\phi}{\longrightarrow}-\alpha_{k}
$$

exchanges $\mathscr{A}$ and $\mathscr{B}$ and leaves $\Omega$ invariant (as a state of $[\mathscr{A} \cup \mathscr{B}]^{=}$). The isomorphism $\phi$ can therefore [6] be implemented by a unitary operator $V$ and extends to the weak closure to give

$$
V \mathscr{A} V^{-1}=\mathscr{B} .
$$

We end this section proving that there exists a unitary operator $U$ with the property

$$
U \mathscr{A}^{1} U^{-1}=\mathscr{B} .
$$

Consider, on $\bigotimes_{i}^{\Omega}\left(\mathscr{H}_{F}^{A_{i}} \otimes \mathscr{H}_{F}^{B_{i}}\right)$ the unitary operators

One checks easily that

$$
U_{i}=\exp \left\{i \pi \sum_{k<i}\left(\alpha_{k}^{*} \alpha_{k}+\beta_{k}^{*} \beta_{k}\right) \beta_{k}^{*} \beta_{k}\right\} \text {. }
$$

$$
\Omega_{i}=\frac{1}{\sqrt{1+\lambda_{i}^{2}}}|0\rangle_{\alpha_{i}}|0\rangle_{\beta_{i}}+\frac{\lambda_{i}}{\sqrt{1+\lambda_{i}^{2}}}|1\rangle_{\alpha_{i}}|1\rangle_{\beta_{i}}
$$

(with obvious notations), and therefore

Therefore the limit

$$
U_{i}\left(\underset{n}{\otimes} \Omega_{n}\right)=\bigotimes_{n} \Omega_{n}
$$

$$
\lim _{i \rightarrow \infty} U_{i}
$$

exists in the weak sense [10], and defines a unitary operator $U$. One easily verifies that $U$ has the property indicated in (20). Combining Eq. (19) and (20) one also obtains

$$
\mathscr{A}=W \mathscr{A}^{\prime} W^{-1}
$$

(weak duality) where $W=V^{-1} U$. 


\section{Free Bose Gas at Finite Temperature}

We shall now consider the structure of the algebra associated [1] to a free bose gas at finite temperature.

The analysis will parallel very closely the one we have given for the free Fermi gas; we shall therefore give only a brief sketch, omitting many details. Also for the Bose gas we shall treat only the case in which there is no condensation in the ground state (physically, temperatures above the critical one). The generalization to include a system with a condensed phase is trivial and leads to no essential change in the structure of the algebra of interest. To fix our notation, we recall that a representation on a Hilbert space $\mathscr{H}$ of the canonical commutation relations (c.c.r.) (a Weyl system) over a Hilbert space $h$ (in what follows, $h=\mathscr{L}_{r}^{2}\left(\mathbf{R}^{n}\right)$ ) is a mapping from $h$ to pairs of unitary operators on $\mathscr{H}$ such that

$$
\begin{aligned}
h, f & \rightarrow\{U(f), V(f)\} \\
U\left(f_{1}\right) V\left(f_{2}\right) & =V\left(f_{2}\right) U\left(f_{1}\right) e^{i\left(f_{1}, f_{2}\right)} \\
{\left[U\left(f_{1}\right), U\left(f_{2}\right)\right]_{-} } & =\left[V\left(f_{1}\right), V\left(f_{2}\right)\right]_{-}=0
\end{aligned}
$$

$V(\lambda f)$ and $U(\lambda f)$ are, for each $f \in h$, weakly continuous in $\lambda$ at zero.

One can define closed operators $a(f), a^{*}(f)$ on a dense set of $\mathscr{H}$, by

$$
\begin{aligned}
& \lim _{\lambda \rightarrow 0} \frac{U(\lambda f)-1}{\lambda}=a(f)+a^{*}(f) \\
& \lim _{\lambda \rightarrow 0} \frac{V(\lambda f)-1}{i \lambda}=a(f)-a^{*}(f) .
\end{aligned}
$$

The $a$, resp. $a^{*}$ are called destruction, resp. creation operators. They satisfy the commutation relations (always on a dense set of vectors)

$$
\begin{aligned}
& {\left[a(f), a^{*}(g)\right]_{-}=(f, g) \quad \forall f, g \in \mathscr{L}_{r}^{2}\left(\mathbf{R}^{n}\right) .} \\
& {[a(f), a(g)]_{-}=0}
\end{aligned}
$$

An irreducible representation of a Weyl systems is called of Fock type if there exists a vector $\Omega \in \mathscr{H}$ in the domain of all $a(f)$ such that

$$
a(f) \Omega=0 \quad \forall f \in h .
$$

Equivalently, $\Omega$ is characterized by

$$
(\Omega U(f) V(g) \Omega)=\exp \left\{-\frac{1}{4}|f|^{2}-\frac{1}{4}|g|^{2}-\frac{i}{2}(f, g)\right\} .
$$

If the representation is of Fock type, we shall write

$$
\mathscr{H}=\mathscr{H}_{F}^{a}, \quad U(f)=U_{F}^{a}(f), \quad V(f)=V_{F}^{a}(f) .
$$

With these notations, a free Bose gas at finite temperature, in absence of condensation and with density $\varrho(k), \varrho>0, k \in \mathbf{R}^{n}$ (interpreted as momentum space), is described [1] by the following representation of the Weyl system over $\mathscr{L}_{r}^{2}\left(\mathbf{R}^{n}\right)$, on $\mathscr{H}_{F^{F}}^{a} \otimes \mathscr{H}_{F^{1}}^{b} \equiv \mathscr{H}_{F}^{a, b}$

$$
\begin{aligned}
& V^{\alpha}(f)=V_{F}^{a}\left((1+\varrho)^{1 / 2} f\right) \otimes V_{F}^{b}\left(-\varrho^{1 / 2} f\right) \\
& U^{\alpha}(f)=U_{F}^{a}\left((1+\varrho)^{1 / 2} f\right) \otimes U_{F}^{b}\left(\varrho^{1 / 2} f\right) .
\end{aligned}
$$


The corresponding creation and destruction operators are written

$$
\begin{aligned}
\alpha(f) & =\underline{a}\left((1+\varrho)^{1 / 2} f\right)+\underline{b}^{*}\left(\varrho^{1 / 2} f\right), \\
\alpha^{*}(f) & =\underline{a}^{*}\left((1+\varrho)^{1 / 2} f\right)+\underline{b}\left(\varrho^{1 / 2} f\right)
\end{aligned}
$$

where, e.g.

$$
\underline{a}(g)=a(g) \otimes \mathbf{I}
$$

$$
\left.a(g) \in \mathscr{L}\left(\mathscr{H}_{F}^{a}\right) \quad \text { (linear operators on } \mathscr{H}_{F}^{a}\right) .
$$

The similarity with (1) is obvious; the analysis of the structure of the algebra $\mathscr{A}$ "generated" by the $\alpha(f), f \in \mathscr{L}^{2}\left(\mathbf{R}^{n}\right)$ will parallel the one given for the Fermi system, with the simplifying features that all injections (e.g. $a(g) \rightarrow \underline{a}(g), b(g) \rightarrow \underline{b}(g))$ will be trivial since

$$
[a(f), b(g)]_{-}=0 \quad \forall f, g .
$$

Define (this parallels $(3)$ )

i.e.

$$
\begin{aligned}
& U^{\beta}(f)=U_{F}^{a}\left(\varrho^{1 / 2} f\right) \otimes U_{F}^{b}\left((1+\varrho)^{1 / 2} f\right), \\
& V^{\beta}(f)=V_{F}^{a}\left(-\varrho^{1 / 2} f\right) \otimes V_{F}^{b}\left((1+\varrho)^{1 / 2} f\right)
\end{aligned}
$$

One checks easily

$$
\beta(f)=\underline{a}^{*}\left(\varrho^{1 / 2} f\right)+\underline{b}\left((1+\varrho)^{1 / 2} f\right) .
$$

$$
\left[U^{\alpha}(f), U^{\beta}(g)\right]_{-}=\left[U^{\alpha}(f), V^{\beta}(g)\right]_{\perp}=\left[V^{\alpha}(f), V^{\beta}(g)\right]=0
$$

i.e.

$$
[\beta(f), \alpha(g)]=\left[\beta(f), \alpha^{*}(g)\right]=0 \quad \forall f, g \in \mathscr{L}_{r}^{2}\left(\mathbf{R}^{n}\right) .
$$

Apply now Lemma 1 , with $A=\sqrt{\frac{\varrho}{1+\varrho}}$. Since $A$ has spectrum in $0 H 1$, also $D$ can be chosen to have spectrum in the same interval. Let $\lambda_{i}$ be the eigenvalues of $D, 0 \leqq \lambda_{i} \leqq 1,\left\{f_{i}\right\}$ the corresponding (orthonormal complete) set of eigenvectors.

i.e.

$$
\begin{aligned}
U^{A_{i}} & =\left(U^{\alpha_{i}}\right)^{c_{i}}\left(U^{\left.\beta_{i}\right)^{s_{i}}}\right. \\
V^{A_{i}} & =\left(V^{\alpha_{i}}\right)^{c_{i}}\left(V^{\beta_{i}}\right)^{-s_{i}} \\
U^{B_{i}} & =\left(U^{\beta_{i}}\right)^{c_{i}}\left(U^{\left.\alpha_{i}\right)^{s_{i}}}\right. \\
V^{B_{i}} & =\left(V^{\beta_{i}}\right)^{c_{i}}\left(V^{\alpha_{i}}\right)^{-s_{i}} \\
c_{i} & =\left(1-\lambda_{i}^{2}\right)^{-1 / 2}, \quad s_{i}=\lambda_{i}\left(1-\lambda_{i}^{2}\right)^{-1 / 2}
\end{aligned}
$$

$$
\begin{aligned}
& A_{i}=c_{i} \alpha_{i}-s_{i} \beta_{i}^{*}, \\
& B_{i}=c_{i} \beta_{i}-s_{i} \alpha_{i}{ }^{*} .
\end{aligned}
$$

Repeating the arguments which led from (7) to (11), one proves that the representation given by the $A_{i}, B_{i} i=1,2, \ldots$ is of Fock type, i.e. there exists $\Omega \in \mathscr{H}_{F}^{a, b}$ such that $A_{i} \Omega=B_{i} \Omega=0$. One can therefore write $\mathscr{H}_{F}^{a, b}=\mathscr{H}_{F}^{A, B}$, where $A\left(f_{i}\right)=A_{i}$. For the algebra $A$ one has again an 
expression like (16)

$$
\mathscr{A} \cdot \equiv\left[\mathbf{I}_{i} \otimes \cdots \otimes\left(\mathscr{B}\left(\mathscr{H}_{F}^{\alpha_{i}}\right) \otimes \mathbf{I}_{i}^{\beta_{i}}\right) \otimes \mathbf{I}_{i+1} \ldots, i=1,2, \ldots\right]^{-}
$$

where $\alpha_{i} \equiv \alpha\left(f_{i}\right)$ and $\mathscr{H}_{F^{i}, i}^{\alpha}$ is an infinite-dimensional separable Hilbert space.

As in the Fermi case (compare Eqs. (19), (20), (21) one can show that there exists a unitary operator $V$ on $\mathscr{H}_{F}^{A}, B$ such that

$$
\mathscr{A}=V \mathscr{B} V^{-1}
$$

where $\mathscr{B}$, the Von Neumann algebra generated by the representation $\beta(f), \beta^{*}(f)$ of the c.c.r., has the form

$$
\mathscr{B}=\left[\mathbf{I}_{i} \otimes \cdots\left(\mathbf{I}_{i}^{\alpha} \otimes \mathscr{B}\left(\mathscr{H}_{F}^{\beta_{i}}\right)\right) \otimes \mathbf{I}_{i+1} \ldots, i=1,2, \ldots\right]^{-} .
$$

One has also the "duality theorem" (notice the simplification as compared to $(20))$

$$
\mathscr{A}=\mathscr{B}^{\prime}
$$

\section{Structure Analysis, Bose Case}

We can now determine the type of the factor $\mathscr{A}$. As in the previous case, it depends on the numerical sequence $\left\{d_{i}\right\}, 0 \leqq d_{i} \leqq 2$, defined by

$$
d_{i}=\inf _{\xi_{i}, \eta_{i}}\left|\Omega-\xi_{i} \otimes \eta_{i}\right|^{2}, \quad \xi_{i} \in \mathscr{H}_{F}^{A_{i}}, \quad \eta_{i} \in \mathscr{H}_{F}^{B_{i}}, \quad\left|\xi_{i}\right|=\left|\eta_{i}\right|=1 .
$$

If $\sum_{i} d_{i}<\infty$ a straightforward application of Lemma 2 shows that the algebra $\mathscr{A}$ is a multiple of the Fock representation and is therefore of type $\mathrm{I}_{\infty}$. In all other cases, $\mathscr{A}$ is of type III. Indeed, one has the following

Lemma 3. If $\sum_{i} d_{i}=\infty, \mathscr{A}$, as defined in (31), is a factor of type III.

The proof of this lemma can be found in ref. (11), see also ref. (4). One can also give a shorter proof, using the results of refs. $[14,15]$.

It remains to be seen under which conditions two algebras, $\mathscr{A}_{1}$ and $\mathscr{A}_{2}$ associated to densities $\varrho_{1}$ and $\varrho_{2}$ are unitarily equivalent (i.e. spatially isomorphic).

Since $\mathscr{A}_{1}$ and $\mathscr{A}_{2}$ are either type III or type $I_{\infty}$ with $I_{\infty}$ commutant, $\mathscr{A}_{1}$ and $\mathscr{A}_{2}$ will be unitarily equivalent if (and only if) they are isomorphic [5]. One can easily verify that in the case we are examining, one has

$$
d_{i}=2\left(1-\sqrt{1-\overline{\lambda_{i}^{2}}}\right) .
$$

The presence of a continuous part in the spectrum of the operator $\sqrt{\frac{\varrho}{1+\varrho}}$ is incompatible with $\sum_{i} \lambda_{i}<\infty$ (see Lemma 1); moreover, all the eigenspaces of $\sqrt{\frac{\varrho}{1+\varrho}}$ to the eigenvalue $c, 0 \leqq c \leqq 1$, are infinite-dimensional. We have therefore 
Proposition 2. a) The Von Neumann algebra $\mathscr{A}$ associated to the description of a Bose gas at finite temperature, with density $\varrho$, is of type I if and only if $\varrho$ is different from zero only on a set of Lebesgue measure zero. In all other cases, $\mathscr{A}$ is a factor of type III.

b) Two algebras $\mathscr{A}_{1}$ and $\mathscr{A}_{2}$ associated with densities $\varrho_{1}, \varrho_{2}$ are unitarily equivalent if and only if they are isomorphic.

c) Two algebras $\mathscr{A}_{1}$ and $\mathscr{A}_{2}$ are isomorphic if ${ }^{6}$ the ranges of the two corresponding density functions $\varrho_{1}, \varrho_{2}$ contain the same open intervals and the same isolated points.

\section{Relativistic Neutral Free Field, Spin Zero}

A relativistic neutral free field $\phi$ with spin zero and mass $m$ is [16] a distribution with values (unbounded) operators on a space $\mathscr{H}$, and satisfying the Klein-Gordon equation. In other words, if $g, \mathbf{f} \in \mathscr{D}_{2}\left(\mathbf{R}^{4}\right)^{7}$ one has

$\phi(\mathbf{f})$ is an unbounded operator on $\mathscr{H}$

$$
\begin{aligned}
& \phi(\lambda \mathfrak{f})=\lambda \phi(\mathfrak{f}) ; \quad \phi\left(\mathbf{f}_{1}+\mathfrak{f}_{2}\right)=\phi\left(\mathfrak{f}_{1}\right)+\phi\left(\mathfrak{f}_{2}\right) \\
& \phi\left(\left(\square+m^{2}\right) \mathfrak{f}\right)=0 \quad \forall \mathfrak{f} ; \quad \square=\frac{\partial^{2}}{\partial x_{4}^{2}}-\sum_{1}^{3} \frac{\partial^{2}}{\partial x_{i}^{2}}
\end{aligned}
$$

and all the $\phi(\mathfrak{f})$ have a common dense domain of definition. For all $\mathfrak{f}$, $\phi(\mathfrak{f})$ is essentially self-adjoint.

It is further assumed that there exists a vector $\Omega \in \mathscr{H}$ such that

$$
\phi(\mathbf{f}) \Omega=0 \quad \text { if }\left.\quad \tilde{\mathbf{f}}(p)\right|_{p^{0}=\sqrt{p^{2}+m^{2}}}=0 .
$$

Clearly $\phi\left(\mathbf{f}_{1}\right)=\phi\left(\mathbf{f}_{2}\right)$ if $\left.\tilde{\mathbf{f}}_{\mathbf{1}}(p)\right|_{p^{2}=m^{2}}=\left.\tilde{\mathbf{f}}_{\mathbf{2}}(p)\right|_{p^{2}=m^{2}}$; this allows an alternative description of the field, at "a fixed time", using canonical fields $\phi_{0}$ and $\pi_{0}$, at $t=0$, considered now as distributions on $\mathscr{D}_{2}\left(\mathbf{R}^{3}\right)$. The connection is given by

where $\left.\tilde{\mathbf{f}}_{h}(p)\right|_{p_{0}=\sqrt{p^{2}+m^{2}}}=h(\vec{p})=\left.\tilde{\mathbf{f}}_{h}(p)\right|_{p_{0}=-\sqrt{p^{2}+m^{2}}}$.

$$
\pi_{0}(h)=\phi\left(\dot{\mathbf{f}}_{h}\right) \phi_{0}(h)=\phi\left(\mathbf{f}_{h}\right), \quad h \in \mathscr{D}\left(\mathbf{R}^{3}\right), \quad \mathbf{f}_{h} \in \mathscr{D}\left(\mathbf{R}^{4}\right)
$$

The vector $\Omega$ satisfies

$$
\left[\phi_{0}\left(\left(-\nabla^{2}+m^{2}\right) h\right)+i \pi_{0}(h)\right] \Omega=0 \quad \forall h \in \mathscr{D}_{\mathbf{2}}\left(\mathbf{R}^{3}\right)
$$

where $\nabla^{2}=\sum_{i=1}^{3} \frac{\partial^{2}}{\partial x_{i}^{2}}$.

It can be shown that the field acts irreducibly on $\mathscr{H} ; \mathscr{B}(\mathscr{H})$ is therefore the smallest Von Neumann algebra $\mathscr{A}\left(\mathbf{R}^{3}\right)$ affiliated with $\phi_{0}(h)$, $\pi_{0}(h)$ (in the sense that it contains all the elements of the spectral families of the essentially self-adjoint operators $\left.\phi_{0}(h), \pi_{0}(h)\right)$.

${ }_{6}^{6}$ These conditions are likely to be also necessary. One would need for the proof the extension to the c.c.r. of results recently obtained by R. Powers [12] for the c.a.r.

? Space of real infinitely differentiable functions with compact support. 
The free field can be alternatively described ("equal time" formulation, see e.g. [6]) as a representation of the canonical commutation relations (i.e. as a Bose system) over $\mathscr{L}^{2}\left(\mathbf{R}^{3}\right)$. The vector $\Omega$ will play the role of "no particle" state and the representation of the c.c.r. at hand will therefore be Fock. To every $f \in \mathscr{S}_{r}\left(\mathbf{R}^{3}\right)$ one associates a pair $U(f), V(f)$ of unitary operators on $\mathscr{H}$, according to:

$$
\begin{aligned}
f \rightarrow & U(f), V(f), \\
& U(f)=U_{F}\left(\omega^{-1 / 2} f\right) \\
& V(f)=V_{F}\left(\omega^{1 / 2} f\right)
\end{aligned}
$$

where $\omega=\left(-\nabla^{2}+m^{2}\right)^{1 / 2}$ and $U_{F}(\xi), V_{F}(\xi), \xi \in \mathscr{S}_{r}\left(\mathbf{R}^{3}\right)$ define the Fock representation of the Bose system over $\mathscr{L}_{r}^{2}\left(\mathbf{R}^{3}\right)$, with $\Omega$ as no-particle vector. The mapping defined in (35) realizes a representation of the c.c.r. over $\mathscr{L}_{r}^{2}\left(\mathbf{R}^{3}\right)$. Indeed, with $f, g \in \mathscr{S}_{r}\left(\mathbf{R}^{3}\right)$ (dense in $\mathscr{L}_{r}^{2}\left(\mathbf{R}^{3}\right)$ ) one has

$$
U(f) V(g)=V(g) U(f) e^{i \pi\left(\omega^{1 / 2} f, \omega^{1 / 2} g\right)}=V(g) U(f) e^{i \pi(f, g)} .
$$

The choise of the representation (35) is, as well known dictated by "covariance" conditions (under the action of the Lorentz group) (see e.g. ref. [19]). Indeed, for this representation the following is true; let

$$
\lim _{\lambda \rightarrow 0} \frac{U(\lambda f)-1}{\lambda} \equiv i \phi_{0}(f), \quad \lim _{\lambda \rightarrow 0} \frac{V(\lambda f)-1}{\lambda}=i \pi_{0}(f) .
$$

Denoting by $\mathbf{f} \in \mathscr{S}_{r}\left(\mathbf{R}^{4}\right)$ any function such that $\left.\tilde{\mathbf{f}}(p)\right|_{p_{0}=\sqrt{p^{2}+m^{2}}}$ $=\left.\tilde{\mathbf{f}}(p)\right|_{p_{0}=-\sqrt{p^{2}+m^{2}}}=\tilde{f}(\vec{p})$ a unique field $\phi(\mathbf{f})$, satisfying $\phi\left(\left(\square+m^{2}\right) \mathbf{f}\right)$ $=0 \forall \mathbf{f} \in \mathscr{S}\left(\mathbf{R}^{4}\right)$ is defined over $\mathscr{S}_{r}\left(\mathbf{R}^{4}\right)$ by $\phi(\mathbf{f})=\phi_{0}(f) ; \phi(\dot{\mathbf{f}})=\pi_{0}(f)$. The field $\phi$ (which can be identified with the one introduced at the beginning of this section) is real, satisfies the Klein-Gordon equation and the "covariance" condition

$$
U(\Lambda) \phi(g) U^{-1}(\Lambda)=\phi(\Lambda g), \quad g \in \mathscr{S}_{r}\left(\mathbf{R}^{4}\right),
$$

where $(\Lambda g)(x) \equiv g\left(\Lambda^{-1} x\right)$ and $U(\Lambda)$ is the representation of the Lorentz group which is defined on $\mathscr{H}$ through (35) [5] from the unitary mapping $g \rightarrow \Lambda g$ in $\mathscr{L}_{r}^{2}\left(\mathbf{R}^{4}\right)$.

One could have chosen instead of (35), the representation

$$
f \rightarrow U_{F}(f), V_{F}(f) .
$$

This would correspond, in the physicist's terminology, to define the field using the Newton-Wigner localization [20], i.e. labelling the points 
in space-time using the Newton-Wigner position operator. One would then loose covariance of the field (and locality, i.e. commutativity at space-like points, whether associated to the same time or not).

\section{Local Rings, "Equal Time" Formulation"}

Here, we are interested in the structure of the algebras associated to open regions $\mathscr{O} \subset \mathbf{R}^{3}$ (local rings at fixed time). The algebra $\mathscr{A}(\mathcal{O})$ is defined as the Von Neumann algebra generated by all the $U(f), V(f)$, $f \in \mathscr{D}_{r}(\mathcal{O})$. Alternatively one can define it as the smallest Von Neumann algebra affiliated to all $\phi_{0}(f), \pi_{0}(f), f \in \mathscr{D}_{r}(\mathcal{O})$. Notice that $\mathscr{H} \equiv \mathscr{H}_{F}\left(\mathbf{R}^{3}\right)$ $=\mathscr{H}_{F}(\mathcal{O}) \otimes \mathscr{H}_{F}(\mathcal{O} \perp)$ where $\overline{(\mathcal{O} \oplus \mathscr{O} \perp)}=\mathbf{R}^{3}$ and $\mathscr{H}_{F}(D)$ is the Fock space of the Bose system over $D$. The Von Neumann algebra generated by all $U_{F}(f), V_{F}(f), f \in \mathscr{D}_{r}(\mathcal{O})$ is therefore $\mathscr{B}\left(\mathscr{L}^{2}(\mathcal{O})\right) \otimes \mathbf{I}$, a type $I_{\infty}$ factor (with $I_{\infty}$ commutant). This in particular determines the structure of the local rings, when local is understood "a la Newton-Wigner". They are all type $I_{\infty}$ factors, with $I_{\infty}$ commutant. We already know [3] that this is not the case for the algebras $\mathscr{A}(\mathcal{O})$ defined above. To determine the structure of $\mathscr{A}(\mathcal{O})$ we proceed as follows, paralleling the analysis given for the free Bose gas at finite temperature. The step of passing from the $\alpha_{s}^{\prime}$ to the $A_{s}^{\prime}, B_{s}^{\prime}$ (Eq. 30) will correspond to writing the $U(f)$, $V(f) ; f \in \mathscr{D}_{r}(\mathcal{O})$ as a function of some $U_{F}\left(\xi_{i}\right), V_{F}\left(\xi_{i}\right)$, where the $\xi_{i}$ will be an orthonormal complete set in some Hilbert space $\mathbf{h}$. The $U_{F}\left(\xi_{i}\right)$, $V_{F}\left(\xi_{i}\right)$ form the Fock representation of a Bose system over $\mathbf{h}$, and this representation is of infinite-direct product type. Such will also be then the representation given by $U(f), V(f)$ if the choice of the $\xi_{i}$ will have been judicious enough. It seems appropriate (we are looking for the analogous of $\alpha_{i}, \beta_{i}, A_{i}, B_{i}$, in Eq. (30)) to focus our attention on four (not orthogonal) subsets of $\mathscr{L}_{r}\left(\mathbf{R}^{3}\right), \mathscr{M}_{i}^{0}, i=1,2,3,4$, defined by

$$
\begin{aligned}
\mathscr{M}_{1}^{0} & =\left\{\omega^{-1 / 2} f, f \in \mathscr{D}_{r}(\mathcal{O})\right\}, \\
\mathscr{M}_{2}^{0} & =\left\{\omega^{1 / 2} f, f \in \mathscr{D}_{r}(\mathcal{O})\right\}, \\
\mathscr{M}_{3}^{0} & =\left\{\omega^{1 / 2} g, g \in \mathscr{D}_{r}(\mathcal{O} \perp)\right\}, \\
\mathscr{M}_{4}^{0} & =\left\{\omega^{-1 / 2} g, g \in \mathscr{D}_{r}(\mathcal{O} \perp)\right\} .
\end{aligned}
$$

Here $\omega$ is the operator which acts as multiplication by $\sqrt{\vec{p}^{2}+m^{2}}$ on the Fourier transformed of $f$; formally $\omega=\sqrt{-\nabla^{2}+m^{2}}, \nabla^{2}=\sum_{i=1}^{3} \frac{\partial^{2}}{\partial x_{i}^{2}}(m$ is the "mass" of the field).

${ }^{8}$ We shall study here only the "equal time" formulation. The covariant formulation, in which local rings are associated to open-regions in $\mathbf{R}^{4}$ (with the Minkowsky metric), can be treated in much the same way. We plan to come back at some other time to the structure of the local rings in the covariant formulation. 
We shall denote by $\mathscr{M}_{i}$ the closure of $\mathscr{M}_{i}^{0}$ in the topology of $\mathscr{L}_{r}^{2}\left(\mathbf{R}^{3}\right)$. If the boundary of $\mathcal{O}$ is sufficiently regular, one has $\mathscr{L}_{r}^{2}\left(\mathbf{R}^{3}\right)=\mathscr{M}_{1} \oplus \mathscr{M}_{3}$ $=\mathscr{M}_{2} \oplus \mathscr{M}_{4}[3]^{9}$.

With out choice of $\omega$, all sets $\mathscr{M}_{i}^{0}$ are mutually disjoint, since $\left(-\nabla^{2}+m^{2}\right)^{1 / 2}$ is an "antilocal" operator [17] in the sense that

$$
\left(-\nabla^{2}+m^{2}\right)^{1 / 2} f=g, \quad f, g \in \mathscr{D}_{r}(\mathcal{O})
$$

implies $f=g=0$ for any open region $\mathcal{O}$ with non empty open complement $\mathcal{O} \perp$. Also, since $\omega$ is closed and $>m, \mathscr{M}_{2}^{0}=\mathscr{M}_{2}, \mathscr{M}_{3}^{0}=\mathscr{M}_{3}$. We shall not use for the time being the explicit form of $\omega$ nor its antilocality. This will allow us to have the structure of $\mathscr{A}(\mathcal{O})$ also for more general situations and for the case in which $\omega$ is local (e.g. for the non-relativistic free field theory, where $\left.\omega=-\frac{\nabla^{2}}{2 m}\right)$ and is local, in that $\omega f \in \mathscr{D}_{r}(\mathcal{O})$ if $f \in \mathscr{D}_{r}(\mathcal{O})$.

The vectors in $\mathscr{M}_{2}$ determine ${ }^{10}$ a linear operator $\varphi$ (unbounded, in general) from a subset of $\mathscr{M}_{1}$, to $\mathscr{M}_{1}^{\perp}\left(\equiv \mathscr{L}^{2}\left(\mathbf{R}^{3}\right) \ominus \mathscr{M}_{1}\right)$. Indeed, every vector $\eta \in \mathscr{M}_{\mathbf{2}}$ admits a unique decomposition:

$$
\eta=\xi+\zeta, \quad \xi \in \mathscr{M}_{1}, \quad \zeta \in \mathscr{M}_{1}^{\perp} .
$$

We want to define $\varphi$ by

$$
\eta=\xi+\varphi \cdot \xi, \quad \varphi \cdot \xi \in \mathscr{M}_{1}^{\perp}, \quad \eta \in \mathscr{M}_{2} \ominus\left(\mathscr{M}_{2} \cap \mathscr{M}_{1}^{\perp}\right) .
$$

This mapping is certainly linear; to show its existence we have to prove that, if $\eta^{\prime \prime}=\xi+\zeta^{\prime}, \eta^{\prime} \in \mathscr{M}_{2} \ominus\left(\mathscr{M}_{2} \cap \mathscr{M}_{1}^{\perp}\right)$, then $\zeta^{\prime}=\varphi \cdot \xi$.

One has $\eta^{\prime}-\zeta^{\prime}=\eta-\zeta$ i.e. $\eta^{\prime}-\eta=\zeta^{\prime}-\varphi \cdot \xi$. But $\mathscr{M}_{1}^{\perp} \ni \zeta^{\prime}$ $-\varphi \cdot \xi$, therefore $\zeta^{\prime}-\varphi \cdot \xi=0$. The operator $\varphi$ is not necessarily densely defined on $\mathscr{M}_{1}$. Indeed, one can check that the closure of its domain of definition is $\mathscr{M}_{1} \ominus\left(\mathscr{M}_{1} \cap \mathscr{M}_{2}^{\frac{1}{2}}\right)$ (in other words, if $\xi_{0} \in \mathscr{M}_{1} \cap \mathscr{M}_{2}^{\frac{1}{2}}$, it cannot be approximated by a sequence of $\xi_{n}$ which can appear in (37) with a suitable choice of $\eta_{n}$ ).

Also, the null space of $\varphi$ is $\mathscr{M}_{1} \cap \mathscr{M}_{2}$; indeed, if $\varphi \cdot \xi=0$, one has $\mathscr{M}_{2} \ni \eta=\xi \in \mathscr{M}_{1}$. One should notice here that, when $\omega$ is anti-local, $\mathscr{M}_{1}^{0} \cap \mathscr{M}_{2}^{0}=\emptyset$, but one can have $\mathscr{M}_{1} \cap \mathscr{M}_{2} \neq \emptyset$. The operator $\varphi$ is closed; indeed, let $\xi_{n} \rightarrow \xi, \varphi \cdot \xi_{n} \rightarrow \zeta$. Then $\xi_{n}-\varphi \cdot \xi_{n}$ converges and its limit, $\eta$, is in $\mathscr{M}_{2}$ (since $\mathscr{M}_{2}$ is closed).

${ }^{9}$ Since $\mathscr{M}_{3} \subset \mathscr{M}_{1}^{\perp}$, the problem is to show that $\mathscr{M}_{1}^{\perp}=\mathscr{M}_{3}$ i.e. that every $\sigma \in \mathscr{L}^{2}(\mathcal{O}) \cap \mathscr{D}_{\omega}^{1 / 2}$ can be approximated by a sequence of functions $\sigma_{n}, \sigma_{n} \in \mathscr{D}(\mathcal{O})$, such that $\omega^{1 / 2}\left(\sigma_{n}-\sigma\right) \rightarrow 0$ (in the topology of $\left.\mathscr{L}^{2}(\mathcal{O})\right)$. This can be proved at least for open regions $\mathcal{O} \subset \boldsymbol{R}^{3}$, such that $\partial \mathcal{O}$ (the boundary of $\mathcal{O}$ ) is piecewise differentiable (see e.g. [3]).

The structure of $\mathscr{A}(\mathcal{O})$ which we shall exhibit holds actually independently of this restriction; only the duality relation and the fact that $\mathscr{A}$ is a factor depend on it.

10 This analysis can be found in Ref. [3]. We give it here for completeness. 
Therefore $\eta=\xi+\zeta$ for some $\eta \in \mathscr{M}_{2}$, and $\zeta=\varphi \cdot \xi$ q.e.d. One can also check without difficulty that the closure of the range of $\varphi$ is

$$
\mathscr{M}_{1}^{\perp} \ominus\left(\left(\mathscr{M}_{2} \cap \mathscr{M}_{1}^{\perp}\right) \oplus\left(\mathscr{M}_{2}^{\perp} \cap \mathscr{M}_{1}\right)\right) .
$$

To semplify the discussion, we introduce now the notation

$$
\begin{aligned}
& \mathscr{N}_{1}=\mathscr{M}_{1} \cap \mathscr{M}_{2}^{\perp}, \\
& \mathscr{N}_{2}=\mathscr{M}_{1}^{\perp} \cap \mathscr{M}_{2}, \\
& \mathscr{N}_{3}=\mathscr{M}_{1} \cap \mathscr{M}_{2}, \\
& \mathscr{N}_{4}=\mathscr{M}_{1}^{\perp} \cap \mathscr{M}_{2}^{\perp}, \\
& \mathscr{N}_{5}=\mathscr{M}_{1} \ominus\left(\mathscr{N}_{1} \oplus \mathscr{N}_{3}\right), \\
& \mathscr{N}_{6}=\mathscr{M}_{2}^{\perp} \ominus\left(\mathscr{N}_{2} \oplus \mathscr{N}_{4}\right) .
\end{aligned}
$$

In what follows we shall be mainly interested in $\varphi_{0}$, the restriction of $\varphi$ to $\mathscr{N}_{5}$. The operator $\varphi_{0}$ is closed, linear, densely defined (on $\mathscr{N}_{5}$ ) with densely defined inverse ( $\varphi_{0}$ has no proper eigenvalue zero!) and with dense range (in $\mathscr{N}_{6}$ ). It admits therefore an adjoint [18], denoted by $\varphi_{0}^{*}$, a densely defined closed linear operator from $\mathscr{N}_{6}$ to $\mathscr{N}_{5}$.

Moreover, the properties of $\varphi_{0}$ guarantee [18] the existence of the polar decomposition

$$
\varphi_{0}=U_{0}\left(\varphi_{0}^{*} \varphi_{0}\right)^{1 / 2}
$$

where $U_{0}$ is a unitary operator which intertwines $\mathscr{N}_{j}$ with $\mathscr{N}_{6}$.

\section{The Structure of the Local Rings}

We are now in position to discuss the explicit form of the algebras associated to a given open region $\mathcal{O} \in \mathbf{R}^{3}$. We assume of course that $\mathcal{O} \perp$ (the interior of the complement of $\mathcal{O}$ in $\mathbf{R}^{3}$ ) is not empty. Otherwise $\mathscr{A}(\mathcal{O})=\mathscr{A}\left(\mathbf{R}^{3}\right)=\mathscr{B}(\mathscr{H})$ since the field $\phi$ is assumed to act irreducibly on $\mathscr{H}$.

Corresponding to the decomposition

$$
\mathscr{L}^{2}\left(\mathbf{R}^{3}\right)=\bigoplus_{n=1}^{6} \mathscr{N}_{i}
$$

one has a decomposition of the Fock representation

$$
U_{F}(f), V_{F}(f) \quad f \in \mathscr{S}\left(\mathbf{R}^{3}\right)
$$

in the direct product of the six representations

$$
U_{F}\left(f^{(i)}\right), V_{F^{\prime}}\left(f^{(i)}\right), \quad f^{(i)} \in \mathscr{N}_{i} i=1 \ldots 6
$$

the $i^{\text {th }}$ acting on $\mathscr{H}_{F}^{i}$, where $\mathscr{H}=\bigotimes_{i=1}^{6} \mathscr{H}_{F}^{i}$. The ring $\mathscr{A}(\mathcal{O})$ is generated, 
we recall, by the representation of the c.c.r. defined by

$$
\begin{gathered}
\{U(f), V(g)\} \quad f, g \in \mathscr{D}(\mathcal{O}), \\
U(f)=U_{H^{\prime}}\left(\omega^{-1 / 2} f\right) ; \quad V(f)=V_{H^{\prime}}\left(\omega^{1 / 2} f\right) .
\end{gathered}
$$

Equivalently, $\mathscr{A}(\mathcal{O})$ can be considered as generated by

$$
\left\{U_{F}(\xi), V_{F}(\eta)\right\}, \quad \xi \in \mathscr{M}_{1}, \eta \in \mathscr{M}_{2} .
$$

Indeed, $\left\{\omega^{-1 / 2} f ; f \in \mathscr{D}(\mathcal{O})\right\}$ is dense in $\mathscr{M}_{1}$ (for the $\mathscr{L}^{\mathbf{2}}\left(\mathbf{R}^{3}\right)$ norm), $\left\{\omega^{1 / 2} f ; f \in \mathscr{D}(\mathcal{O})\right\}$ is dense in $\mathscr{M}_{2}$, and, for the Fock representation

$$
\left\{U_{F}(x), x \in X\right\}^{-}=\left\{U_{F}\left(x^{\prime}\right), x^{\prime} \in X^{\prime}\right\}^{-}
$$

(and the same for $V_{F}$ ) where $X$ and $X^{\prime}$ are both dense in (a subspace of) $\mathbf{h}$, the space over which the representation is taken. The algebra $\mathscr{A}(\mathcal{O})$ factors therefore in the direct product [7]

$$
\mathscr{A}(\mathcal{O})=\left(\bigotimes_{i=1 \ldots 4}^{c} \mathscr{A}^{(i)}(\mathcal{O})\right)^{c} \otimes^{\prime} \mathscr{A}_{0}(\mathcal{O})
$$

where $\mathscr{A}_{0}(\mathcal{O})$ acts on $\mathscr{H}_{F}^{5} \otimes \mathscr{H}_{F}^{6}$.

It is immediately seen that $\mathscr{A}^{(1)}(\mathcal{O})$ and $\mathscr{A}^{(2)}(\mathcal{O})$ are maximal abelian algebras in $\mathscr{H}_{F}^{(1)}, \mathscr{H}_{F}^{(2)}$ respectively. We sketch the proof for $\mathscr{A}^{(1)}(\mathcal{O})$. One has $V_{H^{\prime}}(0)=\mathbf{I}$ and $\mathscr{M}_{2} \cap\left(\mathscr{M}_{1} \cap \mathscr{M}_{2}^{\frac{1}{2}}\right)=\emptyset$. Therefore $\mathscr{A}^{(1)}(\mathcal{O})$ is generated by $\left\{U_{F}(\xi) ; \xi \in \mathscr{M}_{1} \cap \mathscr{M}_{2}^{\frac{1}{2}}\right\}$, and this is a maximal abelian algebra. The algebra $\mathscr{A}^{(4)}(\mathcal{O})$ is composed of the multiples of the identity in $\mathscr{H}_{F}^{(4)}$, i.e. $\left\{c \cdot \mathbf{I}^{4}\right\}$. Indeed, $\left(\mathscr{M}_{1}^{\perp} \cap \mathscr{M}_{2}^{\perp}\right) \cap \mathscr{M}_{1}=\emptyset=\left(\mathscr{M}_{1}^{\perp} \cap \mathscr{M}_{2}^{\perp}\right) \cap \mathscr{M}_{2} ;$ therefore $\mathscr{A}^{(4)}(\mathcal{O})$ is generated by $U_{F}(0), V_{F}(0)$, i.e. by $\mathbf{I}^{(4)}$. The algebra $\mathscr{A}^{(3)}(\mathcal{O})$ is associated to the Fock representation of the c.c.r. in $\mathscr{H}_{F}^{(3)}$, therefore $\mathscr{A}^{(3)}(\mathcal{O})=\mathscr{B}\left(\mathscr{H}_{F^{\prime}}^{(3)}\right)$. To see this, it is enough to notice that, if $\zeta_{i}$ is an orthonormal complete basis in $\mathscr{M}_{1} \cap \mathscr{M}_{2}, \mathscr{A}(\mathcal{O})$ is generated by $\left\{U_{F}\left(\zeta_{i}\right)\right.$, $\left.V_{F^{\prime}}\left(\zeta_{i}\right)\right\}$, i.e. by $\left\{U_{F}\left(\zeta_{i}\right), V_{F}\left(\zeta_{i}\right)\right\} \forall i$.

One can therefore write

$$
\mathscr{A}(\mathcal{O})=\mathscr{C}_{1} \otimes \mathscr{C}_{2} \otimes \mathscr{B}\left(\mathscr{H}_{H^{\prime}}^{(3)}\right) \otimes\left\{c \mathbf{I}_{F^{\prime}}^{(4)}\right\} \otimes \mathscr{A}_{0}(\mathcal{O})
$$

where $\mathscr{C}_{1}, \mathscr{C}_{2}$ are maximal abelian.

It remains to be determined the structure of $\mathscr{A}_{0}(\mathcal{O})$. From (41) one sees that $\mathscr{A}_{0}(\mathcal{O})$ is generated by

$$
\left\{U_{H^{r}}(\xi), V_{F}\left(\xi+\varphi_{0} \cdot \xi\right)\right\}, \quad \xi \in \mathscr{N}_{5} \cap D \varphi_{0} .
$$

Consider now the algebra $\mathscr{B}(\mathcal{O})$ defined in the same way as $\mathscr{A}(\mathcal{O})$ (see Eq. (41)) but now with $\xi \in \mathscr{M}_{2}^{\perp}, \eta \in \mathscr{M}_{1}^{\perp}$; i.e. $\mathscr{B}(\mathcal{O})$ is generated by

$$
\left\{U_{F}(\xi), V_{F}(\eta)\right\}, \quad \xi \in \mathscr{M}_{2}^{\perp}, \eta \in \mathscr{M}_{1}^{\perp} .
$$

One checks without difficulty that $\mathscr{B}(\mathcal{O})$ decomposes according to

$$
\mathscr{B}(\mathcal{O})=\mathscr{C}_{1} \otimes \mathscr{C}_{2} \otimes\left\{c \cdot \mathbf{I}^{(3)}\right\} \otimes \mathscr{B}\left(\mathscr{H}_{k^{\prime}}^{(4)}\right) \otimes \mathscr{B}_{0}(\mathcal{O}) .
$$

7 Commun. math. Phys., Vol. 9 
The algebra $\mathscr{B}_{0}(\mathcal{O})$ satisfies $\mathscr{B}_{0}(\mathcal{O}) \supset \mathscr{B}_{1}(\mathcal{O})$, where $\mathscr{B}_{1}(\mathcal{O})$ is generated by

$$
\left\{U_{F}\left(\eta-\varphi_{0}^{*} \eta\right), V_{F}(\eta)\right\}, \quad \eta \in \mathscr{N}_{6} \cap D_{\varphi^{*}}
$$

To see this one has only to recall that

$$
\eta-\varphi_{0}^{*} \eta \in \mathscr{M}_{2}^{\perp} \Theta\left(\left(\mathscr{M}_{2}^{\perp} \cap \mathscr{M}_{1}^{\perp}\right) \oplus\left(\mathscr{M}_{2}^{\perp} \cap \mathscr{M}_{1}\right)\right) .
$$

One could show at this point that

$$
\left\{\eta-\varphi_{0}^{*} \eta ; \eta \in D_{q_{0}^{*}}^{*}\right\}^{-}=\mathscr{M}_{2}^{\perp} \Theta\left(\left(\mathscr{M}_{2}^{\perp} \cap \mathscr{M}_{1}^{\perp}\right) \oplus\left(\mathscr{M}_{2}^{\perp} \cap \mathscr{M}_{1}\right)\right)
$$

and therefore $\mathscr{B}_{0}(\mathcal{O})=\mathscr{B}_{1}(\mathcal{O})$.

We shall see this more directly in a moment.

The next step is to construct a new representation of the c.c.r. on $\mathscr{H}_{F^{\prime}}^{(5)} \otimes \mathscr{H}_{F^{\prime}}^{(6)}$ and to show that it is of Fock type.

We shall use, in most of what follows, the notation $\mathscr{H}=\mathscr{H}_{F}^{(5)} \otimes \mathscr{H}_{F}^{(6)}$. Apply Lemma 1 to the self-adjoint operator $\left(\varphi_{0}^{*} \varphi_{0}\right)^{1 / 2}$ on $\mathscr{N}_{5}$. Let $D$ be the corresponding operator with discrete spectrum; let $\xi_{i}, d_{i}$ be the corresponding eigenvectors and eigenvalues. The operator $U_{0}$ is unitary, and therefore the set $U_{0} \xi_{i}$ is orthonormal complete in $\mathscr{N}_{6}$.

The algebras $\mathscr{A}_{0}(\mathcal{O})$ and $\mathscr{B}_{1}(\mathcal{O})$ are given by

$$
\begin{aligned}
\mathscr{A}_{0}(\mathcal{O}) & =\left\{U_{i}^{\alpha}, V_{i}^{\alpha} ; i \in I\right\}, \\
\mathscr{B}_{1}(\mathcal{O}) & =\left\{U_{i}^{\beta}, V_{i}^{\beta} ; i \in I\right\}, \\
U_{i}^{\alpha} & =U_{F}\left(\xi_{i}\right) ; \quad V_{i}^{\alpha}=V_{F}\left(\xi_{i}+\varphi \xi_{i}\right), \\
V_{i}^{\beta} & =V_{F}\left(U_{0} \xi_{i}\right) ; \quad U_{i}^{\beta}=U_{F}\left(U_{0} \xi_{i}-\varphi_{0}^{*} U_{0} \xi_{i}\right)
\end{aligned}
$$

(notice that $\left.\xi_{i} \in D_{\left(\varphi_{0}^{*} \varphi_{0}\right)^{1 / 2}} \cong D_{\varphi_{0}}\right)$.

In terms of creation and destruction operators, one can say that $\mathscr{A}_{0}(\mathcal{O})$ is "affiliated" to the operators

$$
\alpha_{i} \equiv a\left(\xi_{i}+\frac{1}{2} \varphi_{0} \cdot \xi_{i}\right)-\frac{1}{2} a^{*}\left(\varphi_{0} \cdot \xi_{i}\right)
$$

and $\mathscr{B}_{0}(\mathcal{O})$ to the operators

$$
\beta_{i} \equiv a\left(U_{0} \cdot \xi_{i}-\frac{1}{2} \varphi_{0}^{*} U_{0} \cdot \xi_{i}\right)-\frac{1}{2}-a^{*}\left(\varphi_{0}^{*} U_{0} \xi_{i}\right) .
$$

One checks without difficulty that

$$
\left[\alpha_{i}, \alpha_{j}^{*}\right]_{-}=\delta_{i j}=\left[\beta_{i}, \beta_{j}^{*}\right]_{-}
$$

all other pairs commute.

Define now:

$$
\begin{aligned}
U_{i}^{A} & =U_{i}^{\alpha}, \\
V_{i}^{A}=V_{i}^{\alpha}\left(V_{i}^{\beta}\right)^{-\lambda_{i}} & =V_{F}\left(\xi_{i}+\varphi_{0} \cdot \xi_{i}-\lambda_{i} U_{0} \cdot \xi_{i}\right), \\
U_{i}^{B}=U_{i}^{\beta}\left(U_{i}^{\alpha}\right)^{\lambda_{i}} & =U_{F}\left(U_{0} \cdot \xi_{i}-\varphi_{0}^{*} U_{0} \xi_{i}+\lambda_{i} \xi_{i}\right), \\
V_{i}^{B} & =V_{i}^{\beta} .
\end{aligned}
$$


One checks again without difficulty that the $\left\{U_{i}^{A}, V_{i}^{A}\right\}$ and $\left\{V_{i}^{B}, V_{i}^{B}\right\}$ define two representations of the c.c.r.

We set $\varrho_{i} \equiv \varphi_{0} \cdot \xi_{i}-\lambda_{i} U_{0} \xi_{i}=U_{0}\left(\left(\varphi_{0}^{*} \varphi_{0}\right)^{1 / 2}-\lambda_{i}\right) \xi_{i}$.

From Lemma 1 we have

$$
\sum_{i}\left|\varrho_{i}\right|^{2}<\infty
$$

and the argument given in $\S 3$ can be used to conclude that the representations defined by the $\left\{U_{i}^{A}, V_{i}^{A}\right\}$ and $\left\{U_{i}^{B}, V_{i}^{B}\right\}$ are of Fock type.

We can then write

$$
\mathscr{H}=\bigotimes_{i}^{\Omega} \mathscr{H}_{i}, \quad \Omega=\bigotimes_{i} \Omega_{i}, \quad \mathscr{H}_{i}=\mathscr{H}_{F}^{A_{i}} \otimes \mathscr{H}_{F}^{B_{i}}, \quad \Omega_{i}=\Omega_{A_{i}} \otimes \Omega_{B_{i}}
$$

and we have

$$
U_{i}^{A}=\mathbf{I} \otimes \cdots \otimes\left(U^{A_{i}} \otimes \mathbf{I}\right) \otimes \mathbf{I}^{i+1} \otimes \cdots
$$

and similar expressions for $V_{i}^{A}, U_{i}^{B}, V_{i}^{B}$.

Inverting (47), one notices that $U_{i}^{\alpha}, U_{i}^{\beta}, V_{i}^{\alpha}, V_{i}^{\beta}$ are functions of $U_{i}^{A}, U_{i}^{B}, V_{i}^{A}, V_{i}^{B}$ (same index $i$ !). For a finite number of degrees of freedom, every representation is of Fock type. Therefore one can write

$$
\mathscr{H}_{{ }^{i}}^{A_{i}} \otimes \mathscr{H}_{F}^{B_{i}}=\mathscr{H}_{F}^{\alpha_{i}} \otimes \mathscr{H}_{F}^{\beta_{i}}
$$

Relative to the latter decomposition, $\Omega_{i}$ is no longer a product vector. Its minimal distance $d_{i}$ from a (normalized) product vector is seen to be

$$
d_{i}=2\left(1-\frac{1}{\sqrt{1+\lambda_{i}^{2}}}\right)
$$

Since $\left\{U^{\alpha_{i}}, V^{\alpha_{i}}\right\}$ and $\left\{U^{\beta_{i}}, V^{\beta_{i}}\right\}$ act irreducibly on $\mathscr{H}_{F}^{\alpha_{i}}, \mathscr{H}_{F}^{\beta_{i}}$ respectively, we can finally write the expression for $\mathscr{A}_{0}(\mathcal{O}), \mathscr{B}_{0}(\mathcal{O})$ on $\mathscr{N}_{5} \otimes \mathscr{N}_{6}$ as follows where

$$
\begin{aligned}
& \mathscr{A}_{0}(\mathcal{O})=\left\{\mathbf{I} \otimes \cdots\left(\mathscr{B}\left(\mathscr{H}_{i}^{(1)}\right) \otimes \mathbf{I}\right) \otimes \mathbf{I}_{i+1} \ldots, i=1,2, \ldots\right\}^{-}, \\
& \mathscr{B}_{0}(\mathcal{O})=\left\{\mathbf{I} \otimes \cdots\left(\mathbf{I} \otimes \mathscr{B}\left(\mathscr{H}_{i}^{(2)}\right)\right) \otimes \mathbf{I}_{i+1} \ldots, i=1,2, \ldots\right\}^{-}
\end{aligned}
$$

$$
\begin{gathered}
\mathscr{N}_{5} \otimes \mathscr{N}_{6}=\bigotimes_{i}^{\Omega}\left(\mathscr{H}_{i}^{(1)} \otimes \mathscr{H}_{i}^{(2)}\right), \\
\Omega=\bigotimes_{i} \Omega_{i} \quad \Omega_{i} \in \mathscr{H}_{i}^{(1)} \otimes \mathscr{H}_{i}^{(2)}, \\
\inf _{\xi_{i}^{K} \in \mathscr{H}_{i}^{(K)},\left|\xi_{i}^{K}\right|=1}\left|\Omega-\xi_{i}^{1} \otimes \xi_{i}^{2}\right|=2\left(1-\frac{1}{\sqrt{1+\lambda_{i}^{2}}}\right) .
\end{gathered}
$$

One has then $\mathscr{B}_{1}(\mathcal{O})=\mathscr{A}_{0}^{\prime}(\mathcal{O}) \supset \mathscr{B}_{0}(\mathcal{O}) \supset \mathscr{B}_{1}(\mathcal{O})$, and therefore $\mathscr{B}_{0}(\mathcal{O})$ $=\mathscr{B}_{1}(\mathcal{O})$.

From $(50),(43),\left(43^{\prime}\right)$ one sees [10] that $\mathscr{A}(\mathcal{O})$ is of type $I$ if

$$
\sum_{i}\left(1-\frac{1}{\sqrt{1+\lambda_{i}^{2}}}\right)<\infty
$$


of type III otherwise. This means that $\mathscr{A}(\mathcal{O})$ is of type I iff $\varphi^{*} \varphi$ is in trace class or, equivalently, iff $\mathscr{M}_{2}^{(1)} \Theta\left(\mathscr{M}_{2}^{(1)} \cap \mathscr{M}_{1}^{\perp}\right)$ is compact. ${ }^{11}$

We specialize now to the case of interest, namely $\omega=\left(-\nabla^{2}+m^{2}\right)^{1 / 2}$, $m \geqq 0$, and consider only open regions $\mathcal{O} \subset \mathbf{R}^{3}$ with piecewise differentiable boundaries.

In this case, as we have already remarked, one proves [14] that

$$
\begin{aligned}
& \mathscr{M}_{1}^{\perp}=\{\sqrt{\omega} \chi ; \chi \in \mathscr{D}(\mathcal{O})\}^{-}, \\
& \mathscr{M}_{\frac{1}{2}}^{\perp}=\left\{\frac{1}{\sqrt{\omega}} \chi ; \chi \in \mathscr{D}(\mathcal{O})\right\}^{-} .
\end{aligned}
$$

This has the consequence (see $(41),\left(41^{\prime}\right)$ ) that $\mathscr{A}(\mathcal{O} \perp)=\mathscr{B}(\mathcal{O})$. From (43), (43'), (50) and the fact that $\mathscr{C}_{1}, \mathscr{C}_{2}$ are maximal abelian, one derives the duality relation

$$
\mathscr{A}^{\prime}(\mathcal{O})=\mathscr{A}(\mathcal{O} \perp)
$$

From the explicit form of $\mathscr{A}(\mathcal{O})$ one also sees that there exists a unitary operator $V$ such that $\mathscr{A}(\mathcal{O})=V \mathscr{A}(\mathcal{O} \perp) V^{-1}$. Moreover, if $m>0$, the algebra $\mathscr{A}(\mathcal{O})$ is a factor. This amount to say that $\mathscr{M}_{1}^{\perp} \cap \mathscr{M}_{2}=\emptyset$ $=\mathscr{M}_{2}^{\perp} \cap \mathscr{M}_{1}$.

To $\mathscr{M}_{1}^{\perp} \cap \mathscr{M}_{2}=\emptyset$ : notice that $\omega \geqq m>0$ and that $\omega$ is closed. Therefore, if $\xi \in \mathscr{M}_{1}^{\perp} \cap \mathscr{M}_{2}$, there exist $f \in \mathscr{L}^{2}(\mathcal{O}), \chi \in \mathscr{L}^{2}(\mathcal{O} \perp)$ such that $\xi=\sqrt{\omega} f=\sqrt{\omega} \chi$. Multiplying by the (bounded) operator $\omega^{-1 / 2}$ one has $f=\chi$ which implies $f=0, \xi=0$.

To $\mathscr{M}_{1} \cap \mathscr{M}_{2}^{\frac{1}{2}}=\emptyset$; this is equivalent to $\left(\mathscr{M}_{3} \cup \mathscr{M}_{2}\right)^{-}=\mathscr{L}^{2}\left(\mathbf{R}^{3}\right)$. Since $\left\{\omega^{1 / 2} f, f \in \mathscr{S}\left(\mathbf{R}^{3}\right)\right\}$ is dense in $\mathscr{L}^{2}\left(\mathbf{R}^{3}\right)$ (where $\mathscr{S}\left(\mathbf{R}^{3}\right)$ is the Schwartz space of infinitely differ-functions on $\left.\mathbf{R}^{3}\right)$, one has to prove that, for every $g \in \mathscr{S}\left(\mathbf{R}^{3}\right)$, one can find $\varepsilon_{n} \in \mathscr{D}(\mathcal{O}), \delta_{n} \in \mathscr{D}(\mathcal{O} \perp)$ such that

$$
\left|\sqrt{\omega}\left(1-\varepsilon_{n}-\delta_{n}\right) g\right| \underset{n \rightarrow \infty}{\longrightarrow} 0
$$

(the norm being the $\mathscr{L}^{2}\left(\mathbf{R}^{3}\right)$ norm).

One can prove that, if the boundary of $\mathcal{O}$ is piece-wise differentiable, such sequences $\left\{\varepsilon_{n}\right\},\left\{\delta_{n}\right\}$ always exist.

Since $\varepsilon_{n} g \in \mathscr{D}(\mathcal{O}), \delta_{n} g \in \mathscr{D}(\mathcal{O} \perp)$ one concludes that $\mathscr{M}_{3} \cap \mathscr{M}_{2}$ is dense in $\mathscr{L}^{2}\left(\mathbf{R}^{3}\right)$.

One can also prove that $\mathscr{M}_{1}^{\perp} \cap \mathscr{M}_{2}^{\perp}=\emptyset=\mathscr{M}_{1} \cap \mathscr{M}_{2}$. Therefore $\mathscr{A}(\mathcal{O})=\mathscr{A}_{0}(\mathcal{O})$.

Finally from the Lemma 3 one concludes that $\mathscr{A}(\mathcal{O})$ is of type III; indeed, $\varphi_{0}^{*} \varphi_{0}$ cannot be of trace class since the operator $\varphi_{0}$ is unbounded [14]. We summarize the result of this analysis in

Proposition 3. The local ring $\mathscr{A}(\mathcal{O})$ associated, in the fixed-time description of the neutral scalar field, to each region $\mathcal{O} C \mathbf{R}^{3}$ with piece-wise differentiable boundaries, is a hyperfinite type III factor, isomorphic to its

11 In the topology induced by the use, as norm, of the orthogonal distance of $\eta \in \mathscr{M}_{2}^{(1)} \Theta\left(\mathscr{M}_{2}^{(1)} \cap \mathscr{M}_{1}^{\perp}\right)$ from $\mathscr{M}_{1}$. Here $\mathscr{M}_{2}^{(1)}$ is the unit ball of $\mathscr{M}_{2}$. 
commutant, and obtained as direct product of type $I_{\infty}$ factors. Moreover $\mathscr{A}(\mathcal{O} \perp)=\mathscr{A}^{\prime}(\mathcal{O})$ and the factors $\mathscr{A}\left(\mathcal{O}_{1}\right), \mathscr{A}\left(\mathcal{O}_{2}\right)$ are isomorphic if the corresponding operators $\varphi_{1}^{*} \varphi_{1}$ and $\varphi_{2}^{*} \varphi_{2}(E q .(37))$ have the same essential spectrum (i.e. the closures of the two spectra coincide).

\section{Relativistic Free 1/2 Field, Mass Zero Equal Time Formulation}

A relativistic free Fermi field of spin 1/2 and mass zero in the Fock representation is conveniently described at fixed time (see e.g., ref. [19]) by a representation on a Hilbert space $\mathscr{H}$ of the canonical anti-commutation relations over a four-fold copy of $\mathscr{S}\left(\mathbf{R}^{3}\right)$. In our notations, this will correspond to have four types of creation and destruction operators which are distributions on $\mathscr{S}\left(\mathbf{R}^{3}\right)$, and can be physically associated to particle and antiparticle, each existing in one of the two possible (orthogonal) helicity states. More precisely, the field we are considering associates to each function $f \in \mathscr{S}\left(\mathbf{R}^{3}\right) \otimes \mathbb{C}^{4}$ (four-folf copy; $\underline{f} \equiv\left(f_{1}, f_{2}, f_{3}, f_{4}\right)$ ) four creation operators defined by

$$
\begin{aligned}
& \psi_{1}(f)=a_{+}\left(\sqrt{\frac{1+n_{3}}{2}} f\right)+b_{-}^{*}\left(\sqrt{\frac{1-n_{3}}{2}} f\right) \\
& \psi_{2}(f)=a_{+}\left(\frac{n_{1}+i n_{2}}{\sqrt{2\left(1+n_{3}\right)}} f\right)+b_{-}^{*}\left(\frac{n_{1}-i n_{2}}{\sqrt{2\left(1-n_{3}\right.}} f\right) \\
& \psi_{3}(f)=a_{-}\left(\frac{n_{1}+i n_{2}}{\sqrt{2\left(1-n_{3}\right)}} f\right)+b_{+}^{*}\left(\frac{n_{1}+i n_{2}}{\sqrt{1+n_{3}}} f\right) \\
& \psi_{4}(f)=a_{-}\left(\sqrt{\frac{1-n_{3}}{2}} f\right)+b_{+}^{*}\left(\sqrt{\frac{1+n_{3}}{2}} f\right)
\end{aligned}
$$

and the corresponding destruction operators.

The notation used in (51) is:

$$
b^{*}(f)=\left(b\left(f^{*}\right)\right)^{*}, \quad n_{l}=i\left(-\sum_{k} \frac{\partial^{2}}{\partial x_{k}^{2}}\right)^{-1 / 2} \cdot \frac{\partial}{\partial x_{l}}
$$

and e.g. the suffix + in $a_{+}$indicates that $a_{+}$"destroys" a particle of helicity +1 .

It should be noted that, in (51), all operators acting on $f$, are bounded.

One checks without difficulty that

$$
\left[\psi_{i}^{*}(f), \psi_{\imath}(g)\right]_{+}=\delta_{i j}(f, g)
$$

all other anti-commutators vanish.

The algebra $\mathscr{A}(\mathcal{O})$ associated to the region $\mathcal{O} \subset \mathbf{R}^{3}$ is now defined by Eq. (5) when $f \in \mathscr{D}(\mathcal{O})$ (infinitely differentiable functions with support in (0). It should be remarked, as was already done for the relativistic free spin zero field, that this definition of the local field (and of the corresponding local algebra) is the only one compatible with covariance; 
other choices are of course possible, but perhaps less justifiable here, since there exists no "position operator" for a system of mass zero and spin 1/2. As seen from (51), the study of the structure of $\mathscr{A}(\mathcal{O})$ decouples in the study of the (obviously isomorphic) algebras generated by $\psi_{1}(f)$, $\psi_{2}(f)$ and by $\psi_{3}(f), \psi_{4}(f)$ respectively. This is a simplifying feature of the zero-mass case, and justifies treating this particular case first.

We shall denote by $\mathscr{A}_{0}(\mathcal{O})$ the local ring generated by $\psi_{1}(f), \psi_{2}(f)$; one has then, modulo spatial isomorphisms,

$$
\mathscr{A}(\mathcal{O})=\mathscr{A}_{0}(\mathcal{O}) \otimes \mathscr{A}_{0}(\mathcal{O}) \text {. }
$$

It will be expedient to introduce a suitable operator $\varphi$ from $\mathscr{L}^{2}(\mathcal{O}) \otimes \mathbf{C}^{2}$ to $\mathscr{L}^{2}\left(\mathcal{O}^{1}\right) \otimes \mathbf{C}^{2}$. We shall use the following notation.

Two (bounded) operators $E, F$ are defined from $\mathscr{L}^{2}\left(\mathbf{R}^{3}\right) \otimes \mathbf{C}^{2}$ to $\mathscr{L}^{2}\left(\mathbf{R}^{3}\right)$ by

$$
\begin{aligned}
& E \cdot \underline{f}=\sqrt{\frac{1+n_{3}}{2}} f_{1}-\frac{n_{1}+i n_{2}}{\sqrt{2\left(1+n_{3}\right)}} f_{2}, \\
& F \cdot \underline{f}=\sqrt{\frac{1-n_{3}}{2}} f_{1}+\frac{n_{1}+i n_{2}}{\sqrt{2\left(1-n_{3}\right)}} f_{2} .
\end{aligned}
$$

The operator $\varphi$ is then defined by

$$
E \cdot \underline{f}=E \cdot \varphi \cdot f \text {. }
$$

It will be checked now that (54) does indeed define an (unbounded) operator $\varphi$ on a dense subset of $\mathscr{L}^{2}(\mathcal{O}) \otimes \mathbf{C}^{2}$. One has to make sure first that

implies $\underline{g}=0$. Now,

$$
E \cdot \underline{g}=0, \quad \underline{g} \in \mathscr{L}^{2}(\mathcal{O} \perp) \otimes \mathbf{C}^{2}
$$

$$
E \cdot g=\sqrt{\frac{1+n_{3}}{2}} g_{1}-\frac{n_{1}+i n_{2}}{\sqrt{2\left(1+n_{3}\right)}} g_{2},
$$

and this can never vanish, due to the anti-locality of $|i \nabla|$. The proof of this statement is easily obtained using the techniques of ref. [17], and is given explicitely in an appendix. To see that $\varphi$ is densely defined, one notices that, again due to the anti-locality of the $n_{i}$, for a dense set $\{f\}$ in $\mathscr{L}^{2}(\mathcal{O})$ one can find a solution (see appendix) to the equation

$$
\sqrt{\frac{1+n_{3}}{2}} f=\sqrt{\frac{1+n_{3}}{2}} \xi-\frac{n_{1}+i n_{2}}{\sqrt{2\left(1+n_{3}\right)}} \eta ; \quad f \in \mathscr{L}^{2}(\mathcal{O}) ; \xi, \eta \in \mathscr{L}^{2}(\mathcal{O} \perp)
$$

and for another dense set one can find a solution to

$$
\frac{n_{1}+i n_{2}}{\sqrt{2\left(1+n_{3}\right)}} f=\sqrt{\frac{1+n_{3}}{2}} \xi-\frac{n_{1}+i n_{2}}{\sqrt{2\left(1+n_{3}\right)}} \eta \text {. }
$$

Therefore (54) admits a (unique) solution for a dense set in $\mathscr{L}^{2}(\mathcal{O}) \otimes \mathbf{C}^{2}$. The operator $\varphi$ is closed; this follows from the uniqueness of $\varphi$ as defined in (54) and from the fact that $E$ is bounded. 
Again uniqueness of (54) implies that $\varphi$ has no null vector and has dense range; $\varphi^{-1}$ exists and is densely defined on $\mathscr{L}^{2}\left(\mathcal{O}^{\perp}\right) \otimes \mathbf{C}^{2}$.

Let $\varphi^{*}$ be the adjoint of $\varphi$. We want to prove that, on its domain of definition, $\varphi^{*}$ satisfies the equation

where $\boldsymbol{x} \in \mathscr{L}^{2}(\mathcal{O} \perp) \otimes \mathbf{C}^{2}$.

$$
F \cdot \boldsymbol{x}=-F \varphi^{*} \boldsymbol{\chi}
$$

Notice that, for $\mathbf{f}, \mathbf{g} \in \mathscr{L}^{2}\left(\mathbf{R}^{3}\right) \otimes \mathbf{C}^{2}$ one has ${ }^{12}$

$$
(\mathbf{f}, \mathbf{g})=(E \mathbf{f}, E \mathbf{g})+(F \mathbf{f}, F \mathbf{g}) .
$$

Using (56) one can see that, for every $\boldsymbol{\chi} \in D_{\varphi^{*}}$ and $\mathfrak{f} \in D_{\varphi}$

$$
\left(F\left(1+\varphi^{*}\right) \boldsymbol{\chi}, F(1-\varphi) \mathbf{f}\right)=0 .
$$

The proof of $(55)$ is now reduced to showing that $\left\{F(1-\varphi) \mathbf{f} ; \mathbf{f} \in D_{\psi}\right\}$ is dense in $\mathscr{L}^{2}\left(\mathbf{R}^{3}\right)$. Let $(\sigma, F(1-\varphi) \mathbf{f})=0 \forall \mathbf{f} \in D_{\varphi}$. Using the definition of $\varphi$ one obtains

$$
\left(\sigma, \frac{1}{\sqrt{1-n_{3}}}\left(f_{1}-(\varphi \mathbf{f})_{1}\right)\right)=0 \quad \forall \mathbf{f} \in D \varphi .
$$

The set $\left\{f_{1}-(\varphi \cdot \mathfrak{f})_{1} ; \mathbf{f} \in D_{\varphi}\right\}$ coincides with $D_{\left(1-n_{3}\right)^{-1 / 2}}$. As $\left(1-n_{3}\right)^{-1 / 2}$ has dense range, it follows $\sigma=0$ q.e.d.

Since $\varphi$ is densily defined, closed and with a densely defined inverse, one has [18] the polar decomposition

$$
\varphi=U\left(\varphi^{*} \varphi\right)^{1 / 2}
$$

where $U$ is unitary from $\mathscr{L}^{2}(\mathcal{O}) \otimes \mathbf{C}^{2}$ to $\mathscr{L}^{2}(\mathcal{O} \perp) \otimes \mathbf{C}^{2}$.

\section{Structure of the Local Algebras}

Once again, we shall make use of Lemma 1 to approximate $\left(\varphi^{*} \varphi\right)^{1 / 2}$ in the Hilbert-Schmidt norm with an operator $D$ with discrete spectrum. Let $\xi_{i}, \lambda_{i}$ be the eigenfunctions, resp. eigenvalues of $D$. Let $\eta_{i}=U \xi_{i}$. It is seen from (51), (52) that the algebra $\mathscr{A}_{0}(\mathcal{O})$ is generated by the operators $\alpha_{i}, \alpha_{i}^{*}$, where we have chosen

$$
\alpha_{i} \equiv a_{-}\left(E \cdot \xi_{i}\right)+b_{+}^{*}\left(F \cdot \xi_{i}\right)
$$

and the algebra $\mathscr{A}_{0}(\mathcal{O} \perp)$ by the operators $\beta_{i}$, with

$$
\beta_{i} \equiv b_{+}\left(F \cdot \eta_{i}\right)+a_{-}^{*}\left(E \cdot \eta_{i}\right) .
$$

(Recall that the $\xi_{i}$ from an orthonormal complete basis in $\mathscr{L}^{2}(\mathcal{O}) \otimes \mathbf{C}^{2}$ and the $\eta_{i}$ from an orthonormal complete basis in $\mathscr{L}^{2}(\mathcal{O} \perp) \otimes \mathbf{C}^{2}$; and that the weak closure of the Fock representation of a Fermi system does not depend on the particular basis chosen).

${ }^{12}(x, y)$ always stands for the scalar product in the Hilbert space to which $x, y$ belong. 
Introduce now another representation of the c.a.r., defined by the operators

$$
\begin{aligned}
& A_{i}=\frac{1}{\sqrt{1+\lambda_{i}^{2}}}\left(\lambda_{i} \alpha_{i}+\beta_{i}^{*}\right), \\
& B_{i}=\frac{1}{\sqrt{1+\lambda_{i}^{2}}}\left(\lambda_{i} \beta_{i}-\alpha\right) .
\end{aligned}
$$

One has clearly $\left[A_{i}, A_{j}^{*}\right]_{+}=\delta_{i j}=\left[B_{i}, B_{j}^{*}\right]_{+}$, all other anticommutators vanish. In terms of $a_{-}, b_{+}$one has

$$
\begin{aligned}
& A_{i}=\frac{1}{\sqrt{1+\lambda_{i}^{2}}} a_{-}\left(\lambda_{i} E \cdot \xi_{i}+E \cdot \eta_{i}\right)+\frac{1}{\sqrt{1+\lambda_{i}^{2}}} b_{+}^{*}\left(\lambda_{i} F \cdot \xi_{i}+F \cdot \eta_{i}\right), \\
& B_{i}=\frac{1}{\sqrt{1+\lambda_{i}^{2}}} a_{-}^{*}\left(\lambda_{i} E \eta_{i}-E \cdot \xi_{i}\right)+\frac{1}{\sqrt{1+\lambda_{i}^{2}}} b_{+}\left(\lambda_{i} F \cdot \eta_{i}-F \cdot \xi_{i}\right) .
\end{aligned}
$$

Notice that

Therefore

$$
\begin{aligned}
& F \cdot \eta_{i}=-F \cdot \varphi^{*} \eta_{i}=F\left(\varphi^{*} \varphi\right)^{1 / 2} \xi_{i}, \\
& E \cdot \xi_{i}=E \varphi \cdot \xi_{i}=E U\left(\varphi^{*} \varphi\right)^{1 / 2} \xi_{i} .
\end{aligned}
$$

$$
\begin{aligned}
& \sum\left|\lambda_{i} F \cdot \xi_{i}+F \cdot \eta_{i}\right|^{2} \leqq \\
& \Sigma \mid H^{2} \|^{2} \operatorname{Tr}\left(\left(\phi^{*} \varphi\right)^{1 / 2}-D\right)^{2}<\infty, \\
& \sum\left|\lambda_{i} \eta_{i}-E \cdot \xi_{i}\right|^{2} \leqq\|E\|^{2} \operatorname{Tr}\left(\left(\varphi^{*} \varphi\right)^{1 / 2}-D\right)^{2}<\infty .
\end{aligned}
$$

This shows that the representation of the c.a.r. defined on $\mathscr{H}$ by the $A_{i}, B_{i}$, is of Fock type.

One can therefore write the space $\mathscr{H}$ as an infinite (incomplete) direct product $\Theta^{\Omega}\left(\mathscr{H}_{F}^{A_{i}} \otimes \mathscr{H}_{F}^{B_{i}}\right)$ relative to $\Omega$, the "vacuum" of the representation given by $A_{i}, B_{i}$.

From (58) one sees that $\alpha_{i}, \beta_{i}$ are functions of $A_{i}, B_{i}$ (same index $i$ !). On $\mathscr{H}_{F}^{A_{i}} \otimes \mathscr{H}_{F}^{B_{i}}$ the operators $\alpha_{i}, \beta_{i}$ define an irreducible finite-dimensional representation of the c.a.r. One has therefore $\mathscr{H}_{F}^{A_{i}} \otimes \mathscr{H}_{F}^{B_{i}}$ $=\mathscr{H}_{F}^{\alpha_{i}} \otimes \mathscr{H}_{F}^{\beta_{i}}$ and the $\alpha_{i}\left(\right.$ resp. $\beta_{i}$ ) generate a (type $I_{2}$ ) factor isomorphic to $\mathscr{B}\left(H_{F}^{\alpha_{i}}\right)\left(\right.$ resp. $\left.\mathscr{B}\left(H_{F}^{\beta_{i}}\right)\right)$.

The same arguments as given for the free Fermi gas ${ }^{13}$, lead here to the following conclusion, if one recalls the connection between $\mathscr{A}_{0}$ and $\mathscr{A}$ as given by $(52): \mathscr{A}(\mathcal{O})$ can be written as

$$
\mathscr{A}(\mathcal{O})=\left\{\mathbf{I} \otimes \cdots \otimes\left(\mathscr{B}\left(\mathscr{H}_{i}^{(1)}\right) \otimes \mathbf{I}_{i}\right) \otimes \cdots i=1,2 \ldots\right\}^{-}
$$

on $\mathscr{H}=\bigotimes_{i}^{\Omega}\left(\mathscr{H}_{i}^{(1)} \otimes \mathscr{H}_{i}^{(2)}\right), \Omega=\bigotimes_{i} \Omega_{i}, \Omega_{i} \in \mathscr{H}_{i}^{(1)} \otimes \mathscr{H}_{i}^{(2)}$ and $\mathscr{A}(\mathcal{O} \perp)$ is spatially isomorphic to

$$
\mathscr{A}\left(\mathcal{O}^{\perp}\right)=\left\{\mathbf{I} \otimes \cdots \otimes\left(\mathbf{I}_{i} \otimes \mathscr{B}\left(\mathscr{H}_{\imath}^{(2)}\right)\right) \otimes \cdots, i=1,2, \ldots\right\}^{-}
$$

on the same space.

${ }^{1.3}$ And the fact that $\mathscr{B}(\mathscr{H}) \otimes \mathscr{B}(\mathscr{K}) \equiv \mathscr{B}(\mathscr{H} \otimes \mathscr{K})$. 
The algebras $\mathscr{A}(\mathcal{O})$ and $\mathscr{A}(\mathcal{O} \perp)$ satisfy a weak duality, in the sense that there exists a unitary operator $V$ on $\mathscr{H}$ such that

$$
\mathscr{A}(\mathcal{O} \perp)=V \mathscr{A}^{\prime}(\mathcal{O}) V^{-1} \text {. }
$$

As for the type of $\mathscr{A}(\mathcal{O})$, it is of type I if $\sum_{i} d_{i}<\infty$, and of type III otherwise.

Indeed, type $\mathrm{II}_{1}$, which could be possible for an hyperfinite algebra of the type given by Eq. (60), is excluded here by the fact that a local ring can never be of finite type [21].

\section{Relativistic Free Fermi Field, Spin $1 / 2$, Any (Real) Mass}

This slightly more general situation is treated along the lines of the zero-mass case, with some additional technical complications. We shall therefore not bore the reader with detailed computations and shall limit ourselves to a brief sketch.

Let $E, F$ be defined as in $(53)^{14}$ and let $A, B$ be the operators from $\mathscr{K}\left(\mathbf{R}^{3}\right) \equiv \mathscr{L}^{2}\left(\mathbf{R}^{3}\right) \otimes \mathbf{C}^{4}$ to $\mathscr{L}^{2}\left(\mathbf{R}^{3}\right) \otimes \mathbf{C}^{2}$ defined by

$$
B=\left(\begin{array}{ll}
\sqrt{\frac{\omega-m}{2 \omega}} E-\sqrt{\frac{\omega+m}{2 \omega}} E \\
\sqrt{\frac{\omega-m}{2 \omega}} F & \sqrt{\frac{\omega+m}{2 \omega}} F
\end{array}\right) ; A=\left(\begin{array}{ll}
\sqrt{\frac{\omega+m}{2 \omega}} F-\sqrt{\frac{\omega-m}{2 \omega}} F \\
\sqrt{\frac{\omega+m}{2 \omega}} E & \sqrt{\frac{\omega-m}{2 \omega}} E
\end{array}\right)
$$

where $\omega=\left(p^{2}+m^{2}\right)^{1 / 2}, p^{2}=-\nabla^{2}$.

The field relative to spin $1 / 2$, mass $m(m \geqq 0)$ is then defined [29] at equal times associating to each function $f \in \mathscr{K} \oplus \mathscr{K}$ an operator

$$
\psi(f)=a(A f)+b^{*}(B f)
$$

where $a, b$ give two Fock representations over $\mathscr{K}\left(\mathbf{R}^{3}\right)$ on a Hilbert space $\mathscr{H}$.

One has $[a(f), b(g)]_{+}=\left[a(f), b^{*}(g)\right]_{+}=0 \forall f, g \in \mathscr{K}\left(\mathbf{R}^{3}\right) ; \mathscr{B}(\mathscr{H})$ is the smallest Von Neumann Algebra generated by the $\psi(f), f \in \mathscr{K}\left(\mathbf{R}^{3}\right)$. If $\mathcal{O} \subset \mathbf{R}^{3}$, the local ring associated to $\mathcal{O}$ is the algebra generated by all $\psi(f)$, as defined in $(63)$, when $f$ has support in $\mathcal{O}$ (i.e. $f \in \mathscr{K}(\mathcal{O})$ ).

We define now an (unbounded) operator $\varphi$ from a subset of $\mathscr{K}(\mathcal{O})$ to a subset of $\mathscr{K}(\mathcal{O} \perp)$ by

$$
A \cdot f=A \cdot \varphi \cdot f, \quad f \in \mathscr{K}(\mathcal{O}) .
$$

${ }^{14} \mathrm{It}$ should be noted that $\left(\sqrt{1+n_{3}},-\frac{n_{1}+i n_{2}}{\sqrt{1+n_{3}}}\right)$ and $\left(\sqrt{1-n_{3}}, \frac{n_{1}+i n_{2}}{\sqrt{1-n_{3}}}\right)$ are the solutions to the equations $|p|^{-1}(\sigma \cdot p) x=\lambda x$ to the "eigenvalues" $\lambda=+1$ and $\lambda=-1$. 
The reader will have no difficulty in convincing himself that, when $m=0$, the $\varphi$ defined by (64) has the same spectrum as the one introduced using (54).

Again, to show that (64) defines an operator $\varphi$ one has to make sure that $A g=0$ implies $g=0$ if $g \in \mathscr{K}\left(\mathcal{O}^{\perp}\right)$. We shall see that this is indeed the case for any region $\mathcal{O}_{1}$, and, setting $\mathcal{O}_{1}=\mathcal{O}$, we will also have shown that $\varphi^{-1}$ is defined on the range of $\varphi$.

Let $g \in \mathscr{K}\left(\mathcal{O}_{1}\right)$; without loss of generality we shall take $g$ twice differentiable (otherwise we shall consider instead $g^{\prime}=\varepsilon * g$, where $\varepsilon$ is $\mathscr{C}^{\infty}$ and with sufficiently small support and $\varepsilon$ is chosen so that $\varepsilon * g=0$ $\Rightarrow g=0$ and support $g^{\prime}\left(\mathcal{O}_{1}^{\prime}, \mathbf{R}^{3} \ominus \overline{\mathcal{O}_{1}^{\prime}} \neq \emptyset\right)$.

One checks then that $A g=0$ implies

$$
\begin{aligned}
& p_{3} g_{1}+\left(p_{1}+i p_{2}\right) g_{2}=(\omega+m) g_{3} \\
& p_{3} g_{3}+\left(p_{1}+i p_{2}\right) g_{4}=(\omega-m) g_{1}
\end{aligned}
$$

where $g \equiv\left(g_{1}, g_{2}, g_{3}, g_{4}\right), g_{i} \in \mathscr{L}^{2}(\mathcal{O}), p_{k}=i \frac{\partial}{\partial x_{k}} k=1,2,3$. The antilocality of $\omega$, combined with (65), gives $g_{i}=0 i=1 \ldots 4$, i.e. $g=0$ q.e.d. ${ }^{15}$.

Together with the fact that $A$ is bounded, this guarantees also that $\varphi$ is closed.

One proves, much in the same way as for the zero mass case, that $\varphi$ is densely defined and has a dense range. Also, if $\varphi^{*}$ is the adjoint of $\varphi$, one proves that

$$
B \cdot g=-B \cdot \varphi^{*} \cdot g, \quad g \in \mathscr{K}\left(\mathcal{O}^{\perp}\right) \cap D_{\varphi^{*}} .
$$

The proof of this equation is easily adapted from the proof of (55) when one notices that $A^{*} A+B^{*} B=1$ and therefore $(f, g)=(A f, A g)$ $+(B f, B g)$ for every pair $f, g \in \mathscr{K}\left(\mathbf{R}^{3}\right)$. Since $\varphi$ is densely defined, closed and with densely defined adjoint, one has the polar decomposition

$$
\varphi=U\left(\varphi^{*} \varphi\right)^{1 / 2}
$$

where $U$ is unitary from $\mathscr{K}(\mathcal{O})$ to $\mathscr{K}(\mathcal{O} \perp)$.

The analysis of the structure of $\mathscr{A}(\mathcal{O})$ parallels now the one given in $\S 11$; the $\xi_{i}$ will now be an orthonormal complete basis in $\mathscr{K}(\mathcal{O})$ and the $\eta_{i}$ an orthonormal complete basis in $\mathscr{K}(\mathcal{O} \perp)$. The algebra $\mathscr{A}(\mathcal{O})$ is then completely characterized by (compare Eq. (59))

$$
\mathscr{A}(\mathcal{O})=\left\{\mathbf{I} \otimes \cdots \otimes\left(\mathscr{B}\left(\mathscr{H}_{i}^{(1)}\right) \otimes \mathbf{I}_{i}\right) \otimes \cdots i=1,2, \ldots\right\}^{-} .
$$

We can therefore state

${ }^{15} \mathrm{It}$ is instructive to compare this situation with the non-relativistic one. There $A=\left(\begin{array}{ll}F & 0 \\ E & 0\end{array}\right), B=\left(\begin{array}{rr}0 & -E \\ 0 & F\end{array}\right)$. The operator $\varphi$ is then defined only on the vectors $f \in \mathscr{K}$ such that $f_{3}=f_{4}=0$ and is zero on such vectors. This will imply that, in this case, $\mathscr{A}(\mathcal{O})$ is of type $\mathrm{I}$. 
Proposition 4. The local ring $\mathscr{A}(\mathcal{O})$ associated, in the fixed-time description of the free Fermi field of spin $1 / 2$ and mass $m \geqq 0$, to each region $\mathcal{O} \subset \mathbf{R}^{3}$, is a hyperfinite factor, isomorphic to its commutant, and obtained as direct product of type $I_{2}$ factors.

Moreover $\mathscr{A}(\mathcal{O})$ and $\mathscr{A}\left(\mathcal{O}^{\perp}\right)$ are spatially isomorphic, and the operator inducing the isomorphism can be chosen hermitian. Two factors $\mathscr{A}\left(\mathcal{O}_{1}\right)$ and $\mathscr{A}\left(\mathrm{O}_{2}\right)$ are spatially isomorphic if and only if the spectra $\Sigma_{1}, \Sigma_{2}$ of the corresponding operators $\varphi_{1}^{*} \varphi_{1}$ and $\varphi_{2}^{*} \varphi_{\mathbf{2}}$ have the following property: $\boldsymbol{\Xi}_{\mathbf{1}}=\boldsymbol{\Xi}_{\mathbf{2}}$ where $\Xi_{i}, \Xi_{i} \subset[0,1]$, is the closure of the image of $\Sigma_{i}$ under the map

$$
x \rightarrow x, 0 \leqq x \leqq 1, x \rightarrow x^{-1}, 1 \leqq x<\infty .
$$

The factor $\mathscr{A}(\mathcal{O})$ is of type $I$ if $\varphi^{*} \varphi\left(\varphi^{*} \varphi+1\right)^{-2}$ is in trace-class; of type III otherwise.

\section{Relativistic Free Field, Any Mass, Any Half-Integer (Finite) Spin}

We shall now indicate how the analysis developed so far can be applied to determine the structure of the local algebras associated to relativistic free fields with any (positive or zero) mass and finite halfinteger spin. In the following paragraph we shall consider the case of any finite integer spin; similar analysis can of course be carried out for infinite-dimensional (unitary or not unitary) representations of the Lorentz group, but we shall not work them out explicitely here.

A relativistic free field of $\operatorname{spin} s+1 / 2, s$ integer $\geqq 0$, will be described at $t=0$, associating to each $\mathbf{J} \in \mathscr{L}^{\mathbf{2}}\left(\mathbf{R}^{3}\right) \otimes \mathbf{C}^{\mathbf{2}(2 s+2)}$ an operator $\psi(\mathbf{J})$ defined by ${ }^{16}$

$$
\psi(\mathbf{J})=a(A \cdot \mathbf{J})+b^{*}(B \cdot \mathbf{J}) .
$$

Here $A, B$ are continuous operators from $\mathscr{L}^{2}\left(\mathbf{R}^{3}\right) \otimes \mathbf{C}^{2(2 s+2)}$ to $\mathscr{L}^{2}\left(\mathbf{R}^{3}\right) \otimes \mathbf{C}^{2 s+2}$ defined as follows.

Let $\mathbf{J}=\left(\mathscr{J}_{1}, \mathscr{J}_{\mathbf{2}}\right), \mathscr{J}_{i} \in \mathscr{L}^{2}\left(\mathbf{R}^{3}\right) \otimes \mathbf{C}^{2 s+2}$.

Let $u^{k}(\vec{p}) \in \mathscr{L}^{2}\left(\mathbf{R}^{3}\right) \otimes \mathbf{C}^{2 s+2}$ be the normalized solution of the equation

$$
\frac{\vec{\Sigma} \cdot \vec{p}}{|\vec{p}|} u^{k}=k u^{k}, \quad k=s+\frac{1}{2}, \quad s-\frac{1}{2}, \ldots-s-\frac{1}{2}
$$

where $\Sigma_{i} i=1,2,3$ are the generators of the irreducible $(2 s+2)$ dimensional representation of the rotation group.

Let

$u^{(+), k}=\sqrt{\frac{\omega+m}{2 \omega}} u^{k}(i \nabla) ; u^{(-), k}=\varepsilon(k) \sqrt{\frac{\omega-m}{2 \omega}} u^{k}(i \nabla), \varepsilon(k)=\left\{\begin{array}{r}1 k>0 \\ -1 k<0\end{array}\right.$

16 The fleld we shall introduce does not satisfy, for $s>0$, any linear differential equation, for $m \neq 0$. For $m=0, \mathbf{J}$ must be taken in $\mathscr{L}^{2}\left(\mathbf{R}^{3}\right) \otimes \mathbf{C}^{2}$, independently of $s$, and the field satisfies a differential equation of the Weyl type. 
and define

Then $A, B$ are defined by

$$
A_{ \pm}=\left(\begin{array}{l}
u_{1}^{( \pm), s+1 / 2}, \ldots, u_{2 s+2}^{(+), s+1 / 2} \\
\vdots \\
u_{1}^{( \pm),-s-1 / 2}, \ldots, u_{2 s+2}^{(+),-s-1 / 2}
\end{array}\right)
$$

$$
\begin{aligned}
& A \cdot \mathbf{J}=A_{+} \cdot \mathscr{J}_{1}+A_{-} \cdot \mathscr{J}_{2}, \\
& B \cdot \mathbf{J}=-A_{-}^{*} \cdot \mathscr{J}_{1}+A_{+}^{*} \cdot \mathscr{J}_{2} .
\end{aligned}
$$

In $(68),\{a(\mathscr{J})\},\{b(\mathscr{J})\}, \mathscr{J} \in \mathscr{L}^{2}\left(\mathbf{R}^{3}\right) \otimes \mathbf{C}^{2 s+2}$ provide two Fock representations of the c.a.r. ${ }^{17}$ on a Hilbert space $\mathscr{H}$; they satisfy

$$
[a(\mathscr{J}), b(\mathscr{G})]_{+}=\left[a(\mathscr{J}), b^{*}(\mathscr{G})\right]_{+}=0 \quad \forall \mathscr{J}, \mathscr{G}
$$

and the Von Neumann algebra they generate is $\mathscr{B}(\mathscr{H})$. It follows from (68) that $\psi(\mathbf{J})$ satisfies

$$
[\psi(\mathbf{J}), \psi(\mathbf{G})]_{+}=0, \quad\left[\psi(\mathbf{J}), \psi^{*}(\mathbf{G})\right]_{+}=(\mathbf{J}, \mathbf{G}) .
$$

Indeed, one can easily verify that

$$
A^{*} A+B^{*} B=\mathbf{I} \quad \text { on } \quad \mathscr{L}^{2}\left(\mathbf{R}^{3}\right) \otimes \mathbf{C}^{2 s+2} .
$$

At time $t$, the field is defined by

$$
\begin{gathered}
\psi_{t}(\mathbf{J})=\psi(\mathbf{J}(t)), \\
\mathbf{J}(t)=\left(\mathscr{J}_{1}(t), \mathscr{J}_{2}(t)\right), \mathscr{J}_{i}(t)=\int \tilde{\mathscr{J}}_{i}(p) e^{i \vec{p} \cdot \vec{x}-i \omega t} d^{3} p, \quad \omega=\left(p^{2}+m^{7}\right)^{1 / 2} .
\end{gathered}
$$

With these definitions, the distribution-valued field

$$
\psi(\vec{x}, t): \int \psi(\vec{x}, t) \mathbf{J}(\vec{x}) d^{3} x=\psi_{t}(\mathbf{J})
$$

satisfies the Klein-Gordon equation, and is local, in that

$$
\left[\psi(\vec{x}, t), \psi^{*}\left(\vec{y}, t^{\prime}\right)\right]_{+}=0 \quad \text { if } \quad(\vec{x}-\vec{y})^{2}-\left(t-t^{\prime}\right)^{2}>0 .
$$

Eq. (72) can be easily checked, and is due to the fact that, on $\mathscr{L}^{2}\left(\mathbf{R}^{3}\right)$, the operator $\Omega_{t}=\frac{\omega+m}{2 \omega} e^{i \omega t}+\frac{\omega-m}{2 \omega} e^{-i \omega t}$ is "local" in the sense that

$$
\left(\mathscr{G}, \Omega_{t} \mathscr{J}\right)=0 \quad \text { if } \quad d(\operatorname{supp} \mathscr{G}, \operatorname{supp} \mathscr{J})>|t|
$$

where $d\left(\mathcal{O}, \mathcal{O}^{\prime}\right)=\inf _{\substack{\vec{x} \in \Omega \\ \vec{x}^{\prime} \in \Omega}}\left|\vec{x}-\vec{x}^{\prime}\right|$ and $\operatorname{supp} \mathscr{G}$ is the support of the function $\mathscr{G}$.

This "locality" also explains our choice of the operators $A, B$ in (68). We could have chosen instead, e.g., $A^{\prime}, B^{\prime}$, defined by

$$
\begin{aligned}
& A^{\prime} \cdot \mathbf{J}=A \cdot \mathscr{J}_{1}, \\
& B^{\prime} \cdot \mathbf{J}=A \cdot \mathscr{J}_{2} .
\end{aligned}
$$

${ }_{17}$ We choose here to quantize our field according to canonical anticommutation relations. If we do not insist on having positive energy, we can substitute in (68) $b^{*}$ with $b$ and quantize according to canonical commutation relations. We would also in this case see that the local rings are factors of type III and of infinitedirect product type. 
Eq. (70) still holds, but the field $\psi^{\prime}(\mathbf{I})$ is no longer local (in $\mathbf{R}^{4}$ ); the rings associated through the field $\psi^{\prime}$ to each open region $\mathcal{O} \subset \mathbf{R}^{3}$ are of type $\mathrm{I}$.

We are interested in the structure of $\mathscr{A}(\mathcal{O}), \mathscr{O} \subset \mathbf{R}^{3}$, where $\mathscr{A}(\mathcal{O})$ is the Von Neumann algebra generated by $\psi(\mathbf{J}), \mathbf{J} \in \mathscr{L}^{2}(\mathcal{O}) \otimes \mathbf{C}^{2(2 s+2)}$.

The proof that $\mathscr{A}(\mathcal{O})$ is of direct-tensor product type, is now straightforward.

Define a linear operator $\varphi$ from $\mathscr{L}^{2}(\mathcal{O}) \otimes \mathbf{C}^{2(2 s+2)}$ to $\mathscr{L}^{2}(\mathcal{O} \perp) \otimes \mathbf{C}^{2(2 s+2)}$ by

where $A$ is defined in (69). ${ }^{18}$

$$
A \cdot \mathbf{J}=A \cdot \varphi \cdot \mathbf{J}
$$

One checks without difficulty that $\varphi$, as defined in (73), is densely defined on $\mathscr{L}^{2}(\mathcal{O}) \otimes \mathbf{C}^{2(2 s+2)}$, is closed and has dense range in $\mathscr{L}^{2}(\mathcal{O} \perp) \otimes \mathbf{C}^{2(2 s+2)}$. Indeed, $A \cdot \mathbf{J}=0, \mathscr{J} \in \mathscr{L}^{2}(\mathcal{O}) \otimes \mathbf{C}^{2(2 s+2)}$, is seen to imply $\mathbf{J}=0$, since the equation

$$
\sqrt{\frac{\omega+m}{2 \omega}} \mathscr{J}+\sqrt{\frac{\omega-m}{2 \omega}} \mathscr{G}=0, \quad \mathscr{J}, \mathscr{G} \in \mathscr{L}^{2}(\mathcal{O}) \otimes \mathbf{C}^{2 s+2},
$$

implies $\mathscr{J}=\mathscr{G}=0$, due to the antilocality of $\sqrt{\frac{\omega-m}{\omega+m}}$. The operator $\varphi^{*}$ is then defined from a dense subset of $\mathscr{L}^{2}(\mathcal{O} \cdot \mathrm{L}) \otimes \mathbf{C}^{\mathbf{2}(2 s+2)}$ to $\mathscr{L}^{2}(\mathcal{O}) \otimes \mathbf{C}^{2(2 s+2)}$, and, due to $(71)$, is seen to satisfy

$$
B \cdot \boldsymbol{\chi}=-B \cdot \varphi^{*} \cdot \boldsymbol{\chi}, \quad \boldsymbol{\chi} \in \mathscr{L}^{2}(\mathcal{O} \perp) \otimes \mathbf{C}^{2(2 s+2)} .
$$

Once again one considers the self-adjoint operator $\left(\varphi^{*} \varphi\right)^{1 / 2}$, and, in the sense of Lemma 1, an approximating operator $D$ with discrete spectrum. With $\lambda_{i}$ the eigenvalues of $D$, one checks, as in $\S 11$, that $\mathscr{A}(\mathcal{O})$ is of the form

$$
\mathscr{A}(\mathcal{O})=\left\{\mathbf{I} \otimes \cdots \otimes \underset{\otimes \Omega_{i}}{\otimes}\left(\mathscr{B}\left(\mathscr{H}^{i}\right) \otimes \mathbf{I}\right) \otimes \mathbf{I}_{i+\mathbf{1}} \ldots, i=1,2, \ldots\right\}^{-}
$$

on a Hilbert space $\mathscr{H}=\bigotimes_{i}\left(\mathscr{H}^{i} \otimes \mathscr{K}^{i}\right)$ where $\left|\Omega_{i}\right|=1, \mathscr{H}^{i}, \mathscr{K}^{i}$ are twodimensional Hilbert spaces, and

$$
\begin{gathered}
d_{i}=\inf _{\substack{\xi \in H_{i} \\
\eta \in K_{i}}}\left|\xi \otimes \eta-\Omega_{i}\right|, \quad|\xi|=|\eta|=1 \\
\sup \left(\frac{\lambda_{i}}{\sqrt{1+\lambda_{i}^{2}}}, \frac{1}{\sqrt{1+\lambda_{i}^{2}}}\right)=1-\frac{d_{2}}{2} .
\end{gathered}
$$

One can also see that $\mathscr{A}(\mathcal{O})$ and $\mathscr{A}(\mathcal{O} \perp)$ are in "weak duality", in that there exists a unitary operator $V, V^{2}=1$, such that

$$
\mathscr{A}(\mathcal{O} \perp)=V \mathscr{A}^{\prime}(\mathcal{O}) V^{-1} \text {. }
$$

The factor $\mathscr{A}(\mathcal{O})$ is of type I if $\varphi^{*} \varphi\left(\varphi^{*} \varphi+1\right)^{-2}$ is in trace class. It is of type III otherwise.

${ }_{18}$ For $m \neq 0$. When $m=0$, the analysis must be made some-what differently, along the lines of $\S 11$. We shall not repeat it here. 


\section{Relativistic Free Field, Any Mass, Any Integer (Finite) Spin}

As in the preceding paragraph, we shall give a brief sketch of this general case. While for spin zero we restricted ourselves to the neutral case, we shall consider here also charged fields. We shall describe a relativistic "charged" free field, of any mass and spin $s, s$ integer $\geqq 0$, in the following way.

We associate, to each $\mathscr{J} \in \mathscr{S}\left(\mathbf{R}^{3}\right) \otimes \mathbf{R}^{2 s+1}$, two (unbounded) operators on $\mathscr{H}^{19}$

$$
\begin{aligned}
& \phi(\mathscr{J})=a\left(\frac{1}{\sqrt{\omega}} A \mathscr{J}\right)+b^{*}\left(\frac{1}{\sqrt{\omega}} A \mathscr{J}\right), \\
& \pi(\mathscr{J})=i\left[a(\sqrt{\omega} A \mathscr{J})-b^{*}(\sqrt{\omega} A \mathscr{J})\right]
\end{aligned}
$$

where $a, b$ are the "destruction" operators of two Fock representations of the canonical commutation relations, which together generate $\mathscr{B}(\mathscr{H})$. The $(2 s+1)$-dimensional real orthogonal matrix $A$ is defined, in analogy with the half-integer-spin case, by

$$
A=\left(\begin{array}{ccc}
u_{1}^{s} & \ldots & u_{2 s+1}^{s} \\
\vdots & \vdots \\
u_{1}^{-s} & \ldots & u_{2 s+1}^{-s}
\end{array}\right) .
$$

Here $\mathbf{U}^{s}$ is the real solution of the equation

$$
(\vec{\Sigma} \cdot \vec{p}) \mathbf{U}^{s}=s|\vec{p}| \mathbf{U}^{s}
$$

$\left(\Sigma_{k}, k=1,2,3\right.$ are the generators of the irreducible representation of the rotation group with dimension $2 s+1$ ) and one sets, in $(76), p_{k}=i \frac{\partial}{\partial x_{k}}$, $k=1,2,3$.

One extends $\phi, \pi$ to $\mathscr{S}\left(\mathbf{R}^{3}\right)$ by linearity, and to $\mathscr{S}\left(\mathbf{R}^{4}\right)$ defining

$$
\begin{aligned}
& \phi_{t}(\mathscr{J})=a\left(\frac{1}{\sqrt{\omega}} A \mathscr{J}_{t}\right)+b^{*}\left(\frac{1}{\sqrt{\omega}} A \mathscr{J}_{-t}\right), \\
& \pi_{t}(\mathscr{J})=i a\left(\sqrt{\omega} A \mathscr{J}_{t}\right)-i b *\left(\sqrt{\omega} A \mathscr{J}_{-t}\right) .
\end{aligned}
$$

One checks that

$$
\pi_{t}(\mathscr{J})=i \frac{\partial}{\partial t} \phi_{t}(\mathscr{J}), \quad \mathscr{J} \in \mathscr{S}\left(\mathbf{R}^{3}\right) \otimes \mathbf{C}^{2 s+1}
$$

that $\phi_{t}(\mathscr{J})$ is local, since

where

$$
\left[\phi_{t}(\mathscr{J}), \phi_{t^{\prime}}^{*}\left(\mathscr{J}^{\prime}\right)\right]_{-}=0 \text { if } d\left(\mathscr{J}, \mathscr{J}^{\prime}\right)>\left|t-t^{\prime}\right|
$$

$$
d\left(\mathscr{J}, \mathscr{J}^{\prime}\right)=\inf _{\substack{\vec{x} \in \operatorname{supp} J \\ \overrightarrow{x^{\prime}} \in \operatorname{supp} J^{\prime}}}\left|\vec{x}-\vec{x}^{\prime}\right|
$$

and that $\phi(\vec{x}, t)$ defined by $\phi_{t}(\mathscr{J})=\int \mathscr{J}(x) \phi(x, t) d^{3} x$ is a solution of the Klein-Gordon equation to the mass $m$. Equivalently, one can describe

19 The notation used is $a^{*}(\mathscr{J})=\left(a\left(\mathscr{J}^{*}\right)\right)^{*}$. 
the relativistic free field as a Bose system over $\mathscr{L}^{2}\left(\mathbf{R}^{3}\right) \otimes \mathbf{C}^{2 s+1}$ (i.e. a Fock representation of the canonical commutation relations). To each $\mathbf{J} \in \mathscr{S}_{\boldsymbol{R}}\left(\mathbf{R}^{3}\right) \otimes \mathbf{R}^{2(2 s+1)}$ one associates a pair of unitary operators $U(\mathbf{J})$, $V(\boldsymbol{J})$, according to

$$
\begin{aligned}
\mathbf{J} & \rightarrow U(\mathbf{J}), V(\mathbf{J}), \\
U(\mathbf{J}) & =U_{F}\left(\omega^{-\mathbf{1} / \mathbf{2}} \mathbf{A} \cdot \mathbf{J}\right), \\
V(\mathbf{J}) & >V_{F}\left(\omega^{\mathbf{1 / 2}} \mathbf{A} \cdot \mathbf{J}\right)
\end{aligned}
$$

where $U_{F}(\mathbf{J}), V_{F}(\mathbf{J}), \mathbf{J} \in \mathscr{L}_{R}^{2}\left(\mathbf{R}^{3}\right) \otimes \mathbf{R}^{2(2 s+1)}$ define a Fock representation of the Bose system over $\mathscr{L}_{R}^{2}\left(\mathbf{R}^{3}\right) \otimes \mathbf{R}^{2(2 s+1)}$, with $\Omega$ as no-particle state.

$\operatorname{In}(77) \mathbf{A} \cdot \mathbf{J} \equiv\left(A \mathscr{J}_{1} ; A \mathscr{J}_{2}\right)$ where $\mathscr{L}_{R}^{2}\left(\mathbf{R}^{3}\right) \otimes \mathbf{R}^{2(2 s+1)} \ni \mathbf{J}, \mathbf{J}=\left(\mathscr{J}_{1}, \mathscr{J}_{2}\right)$, $\mathscr{J}_{i} \in \mathscr{L}_{R}^{2}\left(\mathbf{R}^{3}\right) \otimes \mathbf{R}^{2 s+1}$, and $A$ is defined in (76). The mapping defined in (78) gives a representation of the canonical commutation relations; one checks indeed that

$$
\begin{aligned}
& U(\mathbf{J}) V(\mathbf{G})=V(\mathbf{G}) U(\mathbf{J}) e^{i \pi(\mathbf{J}, \mathbf{G})}, \\
& U(\mathbf{J}) U(\mathbf{G})=U(\mathbf{J}+\mathbf{G}), \\
& V(\mathbf{J}) V(\mathbf{G})=V(\mathbf{J}+\mathbf{G}) .
\end{aligned}
$$

The explicit relation with the description in terms of $\phi(\mathbf{J}), \pi(\mathbf{J})$ is given by

$$
\begin{aligned}
\lim _{\lambda \rightarrow 0} \frac{U(\lambda \mathbf{J})-I}{i \lambda} & =a\left(\mathscr{J}_{1}\right)+a^{*}\left(\mathscr{J}_{2}\right)+b^{*}\left(\mathscr{J}_{1}\right)+b\left(\mathscr{J}_{2}\right), \\
\lim _{\lambda \rightarrow 0} \frac{V(\lambda \mathbf{J})-I}{\lambda} & =a\left(\mathscr{J}_{1}\right)-a^{*}\left(\mathscr{J}_{2}\right)-b^{*}\left(\mathscr{J}_{1}\right)+b\left(\mathscr{J}_{2}\right), \\
\mathbf{J} & =\left(\mathscr{J}_{1} ; \mathscr{J}_{2}\right) .
\end{aligned}
$$

The local algebra $\mathscr{A}(\mathcal{O})$, associated to a given region $\mathcal{O} \subset R^{3}$, is defined as the Von Neumann algebra generated by $U(\mathbf{J}), V(\mathbf{J}), \mathscr{J}_{i} \in \mathscr{D}_{R}(\mathcal{O}) \otimes \mathbf{R}^{2 s+1}$.

The analysis of the structure of $\mathscr{A}(\mathcal{O})$ is now performed along the lines followed in $\S 9$. The main difference consists in taking functions from $\mathscr{D}(\mathcal{O}) \otimes \mathbf{C}^{2 s+1}$ instead of functions from $\mathscr{D}(\mathcal{O})$. Let $\mathbf{M}_{i}^{0}$, $i=1,2,3,4$ the four subsets of $\mathscr{H}$ defined as in (36), with the substitution $\mathscr{D}(\mathcal{O}) \rightarrow \mathscr{D}(\mathcal{O}) \otimes \mathbf{C}^{2 s+1} ; \mathbf{M}_{i}$ the closure of $\mathbf{M}_{i}^{0}$. Then

$$
\begin{gathered}
\boldsymbol{\eta}=\boldsymbol{\xi}+\varphi \cdot \boldsymbol{\xi}, \\
\boldsymbol{\xi} \in \mathbf{M}_{1} \ominus\left(\left(\mathbf{M}_{1} \cap \mathbf{M}_{2}^{\perp}\right) \oplus\left(\mathbf{M}_{1} \cap \mathbf{M}_{2}\right)\right), \\
\varphi \cdot \boldsymbol{\xi} \in \mathbf{M}_{1}^{\perp} \ominus\left(\left(\mathbf{M}_{1}^{\perp} \cap \mathbf{M}_{2}\right) \oplus\left(\mathbf{M}_{1}^{\perp} \cap \mathbf{M}_{2}^{\perp}\right)\right), \\
\boldsymbol{\eta} \in \mathbf{M}_{2} \ominus\left(\mathbf{M}_{2} \cap \mathbf{M}_{1}^{\perp}\right)
\end{gathered}
$$

defines a closed, unbounded operator $\varphi_{1}$ densely defined and with densely defined inverse. With $\varphi^{*}$ the adjoint of $\varphi$, consider the self-adjoint operator $\varphi^{*} \varphi$ on $\mathbf{M}_{1} \ominus\left(\left(\mathbf{M}_{1} \cap \mathbf{M}_{2}^{\perp}\right) \oplus\left(\mathbf{M}_{1} \cap \mathbf{M}_{2}\right)\right)$. Let $D$ be a self-adjoint operator with discrete spectrum, approximating $\left(\varphi^{*} \varphi\right)^{1 / 2}$ in the sense of Lemma 1. Let $\lambda_{i}$ be the eigenvalues of $D$. As in $\S 9$, one shows that 
$\mathscr{A}(\mathcal{O})$ is of the form

$$
\mathscr{A}(\mathcal{O})=\mathscr{C}_{1} \otimes \mathscr{C}_{2} \otimes \mathscr{B}\left(\mathscr{H}^{(3)}\right) \otimes\left\{c \mathbf{I}^{(4)}\right\} \otimes \mathscr{A}_{0}(\mathcal{O})
$$

where $\mathscr{C}_{1}, \mathscr{C}_{2}$ are maximal abelian and

$$
\mathscr{A}_{0}(\mathcal{O})=\left\{\mathbf{I} \otimes \cdots \otimes\left(\mathscr{B}\left(\mathscr{H}_{i}^{(1)}\right) \otimes \mathbf{I}\right) \otimes \cdots, i=1,2, \ldots\right\}^{-}
$$

acts on $\mathscr{H}^{(5)}=\stackrel{\otimes}{\stackrel{\otimes}{i} \Omega_{i}} \otimes\left(\mathscr{H}_{i}^{(1)} \otimes \mathscr{H}_{i}^{(2)}\right)$ with

$$
2\left(1-\frac{1}{\sqrt{1+\lambda_{i}^{2}}}\right)=\inf _{\xi_{i}^{k} \in \mathscr{H}_{i}^{k}}\left|\Omega-\xi_{i}^{1} \otimes \xi_{i}^{2}\right|, \quad\left|\xi_{i}^{k}\right|=1 .
$$

$\mathscr{A}_{0}(\mathcal{O})$ (and therefore $\mathscr{A}(\mathcal{O})$ ) is of type I if $\sum_{i} \lambda_{i}^{2}<\infty$ (i.e. if $\varphi^{*} \varphi$ is in trace class), of type III otherwise. Using the explicit form of $\omega$, $\omega=\left(-\nabla^{2}+m^{2}\right)$, and considering only open regions $\mathcal{O}$ with differentiable boundaries, one can show, as in $\S 9$, that $\mathscr{A}(\mathcal{O})$ is a hyperfinite factor of type III (since $\varphi$ is unbounded) and that the duality relation holds

$$
\mathscr{A}(\mathcal{O})=\mathscr{A}^{\prime}(\mathcal{O} \perp)
$$

\section{Appendix A}

We shall give here, for completeness, the proof of Lemma 1 [8].

Lemma 1. Let $A$ be a self-adjoint operator on a separable Hilbert space $\mathscr{H}$; then there exists a self-adjoint operator $D$ with discrete spectrum and a Hilbert-Schmidt operator $T$ such that

$$
A=D+T \text {. }
$$

Moreover, one can choose $\|T \mid\|<1$, where $\|T \mid\|$ is the Hilbert-Schmidt norm of $T$.

One establishes first

Lemma 0. With $A$ a self-adjoint operator on $\mathscr{H}, f$ any vector in $\mathscr{H}$ and $\varepsilon>0$, one can find a finite-dimensional projection $P_{f}$ and a HilbertSchmidt self-adjoint operator $T_{f}$, such that $A-T_{f}$ is reduced by $P_{f} \cdot \mathscr{H}$ and $\left|\left(1-P_{f}\right) f\right|<\varepsilon,\left\|\left|T_{f} \|\right|<\varepsilon\right.$.

This lemma is readily proved as follows. Let $A=\int \lambda d E(\lambda)$ be a spectral decomposition of $A$; one can find $a>0$ such that

$$
\left|\left(1-\left(E_{a}-E_{-a}\right)\right) f\right|<\varepsilon .
$$

Divide the interval $-a H+a$ in $n$ disjoint subintervals $\Delta_{i}$ of length $2 a / n$, and let

$$
g_{i}=\frac{E\left(\Delta_{i}\right) f}{\left|E\left(\Delta_{i}\right) f\right|} \quad \text { if } \quad E\left(\Delta_{i}\right) f \neq 0, g_{i}=0 \quad \text { if } \quad E\left(\Delta_{i}\right) f=0 .
$$


Let $P_{f}^{(n)}$ be the orthogonal projection on the subspace spanned by the $g_{i}$; one can verify that

$$
\left|\left(1-P_{f}^{n}\right) f\right|<\varepsilon, \quad \|||\left(1-P_{f}^{n}\right) A P_{f}^{n}|| \mid \leqq \frac{2 a}{n^{1 / 2}}
$$

Taking $n_{0}>\frac{16 a^{2}}{\varepsilon^{2}}$ and $P_{f}=P_{f}^{n_{0}}$, we have $\left|\left(1-P_{f}\right) f\right|<\varepsilon,\left\||| T_{f}\right\|<\varepsilon$ where $T_{f}=\left(1-P_{f}\right) A P_{f}+P_{f} A\left(1-P_{f}\right)$. Obviously $A-T_{f}$ is reduced by $P_{f} \cdot \mathscr{H}$. q.e.d.

To prove now lemma 1 , let $\left\{f_{k}\right\}$ be a Hamel basis in $\mathscr{H}\left(\left\{f_{k}\right\}\right.$ is dense in $\mathscr{H}$ ). Apply lemma 0 to $f_{1}$, with $\varepsilon=1 / 2$ and let $P_{1}, T_{1}$, be the corresponding operators. On $\left(1-P_{1}\right) \mathscr{H}$ apply lemma 0 to the vector $\left(1-P_{1}\right) f_{2}$, and the operator $A_{1} \equiv\left(1-P_{1}\right)\left(A-T_{1}\right)\left(1-P_{1}\right)$, taking now $\varepsilon=2^{-2}$. Extending to $P_{1} \mathscr{H}$ the resulting operators $P_{2}, T_{2}$ by $P_{2} \cdot \xi=T_{2} \cdot \xi=0, \xi \in P_{1} \cdot \mathscr{H}$, one has that $A-T_{1}-T_{2}$ is reduced by $P_{1} \mathscr{H}$ and $P_{2} \mathscr{H}$. By complete induction, one obtains in this way a sequence $\left\{P_{i}\right\}$ of orthogonal projections and a sequence $\left\{T_{i}\right\}$ of selfadjoint Hilbert-Schmidt operators such that

$$
\left|1-\left(P_{1}+\cdots+P_{k}\right)\right| \leqq 2^{-k} ; \quad\left\|\left|T_{k} \|\right| \leqq 2^{-k} .\right.
$$

The series $T_{1}+T_{\mathbf{2}}+\cdots$ converges in the $\||\cdot|\|$ norm; let $T$ be its limit. One has $\|T\| \| \leqq 1$. We prove next that $\sum_{k=1}^{\infty} P_{k}=1$. Let $f \in \mathscr{H}$, $|f|=1$; given $\delta_{0}>0$, one can find $f_{k}$ such that $\left|f-f_{k}\right|<\delta_{0}$; then

$$
\left|\left(1-\sum_{1}^{n} P_{k}\right) f\right| \leqq\left|\left(1-\sum_{1}^{n} P_{k}\right) f_{n}\right|+\delta_{0} \leqq 2^{-n}+\delta_{0} \text {. }
$$

Since $\delta_{0}$ is arbitrary, $\left(1-\sum_{1}^{\infty} P_{k}\right) f=0, \forall f \in \mathscr{H}$ q.e.d.

To show that $P_{k}$ reduces $A-T$, notice that $\left(1-\left(P_{1}+\cdots+P_{n-1}\right)\right) \mathscr{H}$ reduces $A-\left(T_{1}+\cdots+T_{n}\right)$; therefore $P_{n} \mathscr{H}$ reduces $A-T$, since $P_{n} T_{k}=T_{k}=T_{k} P_{n}=0$ for $k>n$.

It remains to be proved that $A-T$ has pure point spectrum. Since $P_{n} \mathscr{H}$ reduces $A-T$ and $P_{n} \mathscr{H}$ is $n$-dimensional, there exist $n$ orthogonal eigenvectors of $A-T$ which form a basis in $P_{n} \mathscr{H}$. Since $\sum_{n=1}^{\infty} P_{n}=1$, the totality of these vectors provides a complete orthonormal basis in $\mathscr{H}$, q.e.d.

The eigenvalues of $A-T$ form a dense set in the spectrum of $A$. This follows because if $A$ is closed and $T$ is Hilbert-Schmidt, $A$ and $A-T$ have the same essential spectrum; it can also be read off easily from the proof, when one notices that the $g_{i}^{(k)}, i=1 \ldots n_{k}$, which are obtained 8 Commun. math. Phys., Vol. 9 
applying the induction procedure outlined above, form are orthonormal complete basis (by construction), and are "approximate eigenfunctions" of $A$, in the sense that

$$
\left|\left(A-\lambda_{i}^{(k)}\right) g_{i}^{(k)}\right|<\frac{a^{(k)}}{n^{(k)}} .
$$

\section{Appendix B}

We want to prove here some of the anti-locality properties of the operators $\sqrt{1+n_{3}}, \frac{n_{1}+i n_{2}}{\sqrt{1+n_{3}}}$ which appear in the structure of a relativistic free Fermi field of spin $1 / 2$ and mass zero. We shall follow very closely the treatment in ref. [17]. We show first that, if $f, g \in \mathscr{L}^{2}(\mathcal{O}), \mathcal{O}$ any open region in $R^{3}$ with non-empty open complement $\mathcal{O} \perp$, then

Here

$$
\frac{n_{1}+i n_{2}}{\sqrt{1+n_{3}}} f+\sqrt{1+n_{3}} g=0 \text { implies } f=g=0 .
$$

$$
n_{k}=\frac{p_{k}}{|\vec{p}|}, \quad|\vec{p}|=\left(\sum_{k=1}^{3} p_{k}^{2}\right)^{1 / 2}, \quad p_{k}=i \frac{\partial}{\partial x_{k}} .
$$

It is obviously sufficient to give the proof when $\mathcal{O} \perp$ is a neighborhood of the origin and is rotationally invariant. We can also assume that $f, g$ are infinitely differentiable with derivatives in $\mathscr{L}^{2}(\mathcal{O})$. Indeed, one could otherwise consider the functions $\varepsilon * f, \varepsilon * g$, where $\varepsilon$ is a $C^{\infty}$ function with support in a neighborhood of the origin and $*$ indicates convolution; the new functions have the desired properties and $\varepsilon * g=0, \varepsilon * f=0$ imply $g=f=0$ if $\varepsilon$ has been chosen properly.

The functions $f, g$, are then in the domain of $|\vec{p}|$ and one has

$$
\left(i \frac{\partial}{\partial x_{1}}-i \frac{\partial}{\partial x_{2}}\right) f=|\vec{p}| g+i \frac{\partial}{\partial x_{3}} g
$$

and this can be written

$$
|\vec{p}| g=f \quad g, f \in \mathscr{L}^{2}(\mathcal{O}) .
$$

We have to prove that (B-3) implies $f=g=0$.

The proof is given first for the case $g, f \in \mathscr{L}^{2}\left(\mathbf{R}^{1}\right)$ and then extended to $f, g \in \mathscr{L}^{2}\left(\mathbf{R}^{3}\right)$, In one dimension,

where

$$
|p|=\varepsilon(p) \cdot p
$$

$$
\varepsilon(p)=\left\{\begin{array}{rl}
1 & p>0 \\
-1 & p<0
\end{array}\right.
$$

Since $g(x)=0,|x|<\delta$ (we take $\mathcal{O} \perp:\{x,|x|<\delta\}$ ) and $g \in \mathscr{L}^{2}\left(\mathbf{R}^{1}\right)$ one can write

$$
(|p| g)(x)=\lim _{\lambda \rightarrow 0_{+}} F_{1}(x+i \lambda)+\lim _{\lambda \rightarrow 0_{-}} F_{2}(x+i \lambda)
$$


where

$$
\begin{aligned}
& F_{1}(z)=\int \frac{\frac{\partial}{\partial x^{\prime}} g\left(x^{\prime}\right)}{z-x^{\prime}} d x^{\prime}, \quad \operatorname{Im} z>0, \\
& F_{2}(z)=\int \frac{\frac{\partial}{\partial x^{\prime}} g\left(x^{\prime}\right)}{z-x^{\prime}} d x^{\prime}, \quad \operatorname{Im} z<0
\end{aligned}
$$

$F_{1}$ is analytic in the half-plane $\operatorname{Im} z>0, F_{2}$ in the half-plane $\operatorname{Im} z<0$. Since $g(x)=0$ when $|x|<\delta, F_{1}(z)$ and $F_{2}(z)$ describe a single function $F(z)$, analytic in the whole complex plane, with the exception of the "cuts" $\operatorname{Im} z=0,|\operatorname{Re} z|>\delta$. Eq. (B-4) implies $F(z)=0$ for $\operatorname{Im} z=0$, $|\operatorname{Re} z|<\delta$. Since $F(z)$ is analytic there, it must be $F(z) \equiv 0$, which in turn implies $f=g=0$ in (B-3).

We extend now the proof to the rotationally symmetric functions on $\mathbf{R}^{3}$. Denote by $\mathscr{J}_{1}, \mathscr{J}_{3}$ the Fourier transform mappings in $\mathscr{L}^{2}\left(\mathbf{R}^{1}\right)$, $\mathscr{L}^{2}\left(\mathbf{R}^{3}\right)$ respectively; let $K$ be the mapping from the rotationally invariant functions on $\mathbf{R}^{3}$ to the symmetric functions on $\mathbf{R}^{1}$ defined by

Then one has

$$
K f=F ;\left.\quad F(\varrho)\right|_{\varrho=\sqrt{\sum_{i} x_{i}^{2}}}=f(\vec{x}) .
$$

where

$$
\frac{1}{2} Q=\mathscr{J}_{1}^{-1} Q K \mathscr{J}_{3}
$$

Therefore

$$
Q f(v) \equiv v f(v)
$$

$$
\left|i \frac{\partial}{\partial q}\right| q K f=\sqrt{\sum_{i} q_{i}^{2}}|p| f \quad|p|=\left(-\nabla^{2}\right)^{1 / 2} .
$$

Let $f(x)=|p| f(x)=0, x \in \mathcal{O}^{\perp}$, a symmetric neighborhood of the origin in $\mathbf{R}^{3}$.

Then $q K f$ and $\left|i \frac{\partial}{\partial q}\right| q K f$ are both zero on a neighborhood $\Gamma$ of the origin in $\mathbf{R}^{1}$; we have already seen that this implies

$$
q K f=0 \text { i.e. } K f=0, f=0 .
$$

Finally, if $f$ is not rotationally invariant, define for each $x \in \mathbf{R}^{3}$ a function $f_{x}(r)$, on $\mathbf{R}^{1}$, symmetric, by $f_{x}(r) \equiv \int f(\vec{x}+r \vec{\omega}) d \Omega \omega,|\vec{\omega}|=1$. Clearly one can choose $\varepsilon, \delta>0$ such that, if $|x|<\delta, f_{x}(r)=0$ when $|r|<\varepsilon$. Also $\left|i \frac{\partial}{\partial r}\right| f_{x}(r)=\left|-\nabla_{x}^{2}\right| f_{x}(r)$; therefore, if $|x|<\delta, \quad\left|i \frac{\partial}{\partial r}\right| f_{x}(r)=0$ when $|r|<\varepsilon$.

It follows then, by previous results

and therefore $f(x)=0$.

$$
f_{x}(r)=0, \text { for }|x|<\delta
$$


As a corollary of these results, one obtains that the vectors of the form

$$
f+\frac{n_{1}+i n_{2}}{1+n_{3}} g, \quad f, g \in \mathscr{L}^{2}(\mathcal{O})
$$

form a dense set in $\mathscr{L}^{2}(\mathcal{O} \perp)$.

Indeed, $\frac{p_{1}+p_{2}}{|p|+p_{3}} g=-f+\sigma, f \in \mathscr{L}^{2}(\mathcal{O}), \sigma \in \mathscr{L}^{2}(\mathcal{O} \perp)$ for all $g$ in the domain of $\frac{n_{1}+i n_{2}}{1+n_{3}}$. The $\sigma$ 's so defined form a linear subset of $\mathscr{L}^{2}\left(\mathcal{O}^{\perp}\right)$. If it were not dense, one could find $v \in \mathscr{L}^{2}(\mathcal{O} \perp),\left(\nu, \frac{n_{1}+i n_{2}}{1+n_{3}} g\right)=0 \forall g$. Taking $g=\left(p_{1}-i p_{2}\right) g_{1}, \quad\left(\sqrt{1-n_{3}} v, g\right)=0 \quad \forall g \in \mathscr{L}^{2}(\mathcal{O})$. Therefore $\left.\sqrt{1-n_{3}} v \in \mathscr{L}^{2}(\mathcal{O} \perp), v \in \mathscr{L}^{2}(\mathcal{O}\lrcorner\right)$ i.e. $v=0$ q.e.d.

Acknowledgments. The analysis presented here was initiated while the author was a visiting member at the Institute for Advanced Studies, Princeton, and was essentially completed during a short permanence at the Institut des Hautes Etudes Scientifiques, Bures-sur-Yvette. The author is pleased to record here his gratitude to profs. C. Kaysen and L. Motchane for the warm hospitality.

\section{References}

1. Araki, H., and E. Woods: Representations of the C.C.R. for a nonrelativistic infinite free Bose gas. J. Math. Phys. 4, 637-662 (1963).

2. - , and W. WYss: Representations of canonical anticommutation relation. Helv. Phys. Acta 37, 136-159 (1964).

3. - Von Neumann algebras of local observables for the free scalar field. J. Math. Phys. 1, 1-13 (1964).

4. - Type of Von Neumann algebras associated to the free scalar field. Progr. Theoret. Phys. 32, 956-961 (1964).

5. Diximier, J.: Les algèbres d'operateurs dans l'espace Hilbertien. Paris: Gauthier-Villars 1957.

6. Segal, I.: Mathematical problems of relativistic physics. Am. Math. Soc. Publications, Providence (1963).

7. Guichardet, M. A.: Produits tensoriels infinis. Ann. Ecole Norm. Super. 83, 1-15 (1966).

8. Von Neumann, J.: Charakterisierung des Spektrums eines Integral Operators. Actualités Scient. et Ind. No. 229 (1935).

9. - On infinite direct products. Comp. Math. 6, 1-77 (1938).

10. - Rings of operators III. Ann. Math. 41, 94-162 (1940).

11. Bures, D.: Certain factors constructed as infinite tensor products. Com. Math. 15, 169-191 (1963).

12. Powers, R.: Representation of uniformly hyperfinite algebras. (To be published).

13. Segal, I.: A class of operator algebras which are determined by groups. Duke Math. Journal 18, 221-265 (1951).

14. STöRMER, E.: Types of invariant algebras associated with extremal invariant states. (To be published.)

15. Doplicher, S., D. Kastler, and D. Robinson: Covariance algebras. Commun. Math. Phys. 3, 1-28 (1966). 
16. Jost, R.: General theory of quantized fields. Am. Math. Soc. Publications, Providence (1966).

17. Segal, I., and R. Goodman: Anti-locality of certain invariant operators. J. Math. Mech. 14, 629-638 (1965).

18. Dunford, N., and J. Schwartz: Linear operator, part II. XII, 7, 7. New York: Interscience Publishers 1958.

19. Schweber, S.: Relativistic quantum field theory. Evanston: Row Peterson \& Co. 1961.

20. Newton, R., and E. Wigner: Localized states of elementary systems. Rev. Mod. Phys. 21, 400-406 (1949).

21. KaDison, R.: Types of Von Neumann algebras in quantum field theory. J. Math. Phys. 4, 1511-1517 (1963).

G. F. Dell'Antonio

Istituto di Fisica Teorica

Università di Napoli

Mostra d'Oltremare, Pad. 19

Napoli, Italia 